\title{
Hecke structure on Bredon cohomology
}

\author{
by
}

Jolanta Słomińska (Toruń)

\begin{abstract}
We construct a Hecke structure on equivariant Bredon cohomology with local coefficients and then describe some of its properties. We compare this structure with the Mackey structure defined by T. tom Dieck and with the Illman transfer.
\end{abstract}

0. Introduction. Let $G$ be a finite group. We shall denote by $\mathbb{H}_{G}$ the category whose objects are the $G$-orbits $G / H$, where $H$ is a subgroup of $G$, and whose morphisms are the $\mathbb{Z}(G)$-homomorphisms of the permutation $\mathbb{Z}(G)$-modules $\mathbb{Z}\left(G / H^{\prime}\right) \rightarrow \mathbb{Z}(G / H)([4]$, I.3). A Hecke functor is an additive contravariant functor $T: \mathbb{H}_{G}^{\text {op }} \rightarrow \mathrm{Ab}$, where $\mathrm{Ab}$ is the category of Abelian groups. The category of Hecke functors will be denoted by $\left(\mathbb{H}_{G}^{\mathrm{op}}, \mathrm{Ab}\right)$. The Hecke functors can be considered as cohomological $G$-functors (see [6], [14] and [16]). Their properties are described in [13] and [15]. Every Hecke functor is a Mackey functor.

In this paper, we shall study connections of Hecke functors with Bredon cohomology theory ([2]). Bredon cohomology is an equivariant singular cohomology theory, which is defined on the category $G$-CW of $G$-CWcomplexes. Its coefficients are contravariant functors $M: O_{G}^{\text {op }} \rightarrow \mathrm{Ab}$, where $O_{G}$ is the category with the same objects as $\mathbb{H}_{G}$, whose morphisms are the $G$-maps $G / H^{\prime} \rightarrow G / H$. These functors are called generic $G$-coefficient systems. The category $O_{G}$ can be considered as a subcategory of $\mathbb{H}_{G}$ so for any Hecke functor $T$ and $G$-CW-complex $K$ we have the Bredon cohomology $H_{G}^{*}(K, T)$.

It is well known that the Bredon cohomology $H_{G}^{*}(K, M)$ of a $G$-CWcomplex $K$ with respect to a coefficient system $M$ can be extended to a coefficient system $\mathcal{H}_{G}^{*}(K, M)$ by defining

$$
\mathcal{H}_{G}^{*}(K, M)(G / H)=H_{G}^{*}(G / H \times K, M)=H_{H}^{*}\left(K, M I_{H}\right),
$$

where $I_{H}$ denotes the natural functor $O_{H} \rightarrow O_{G}$ such that $I_{H}\left(H / H^{\prime}\right)=$ $G / H^{\prime}$ whenever $H^{\prime}$ is a subgroup of $H$.

If $M$ is a Mackey functor, then $\mathcal{H}_{G}^{*}(K, M)$ can also be extended to a 
Mackey functor and this definition gives us a Mackey structure on Bredon cohomology with coefficients in $M$, in the sense of [5]. We show that if $M$ is a Hecke functor, then $\mathcal{H}_{G}^{*}(K, M)$ can also be considered as a Hecke functor. This Hecke structure can be considered as an extension of the Mackey structure defined by T. tom Dieck.

In Section 1 we present another extension of $H_{G}^{*}(K, M)$ to a certain coefficient system. We define a Hecke functor $\mathcal{I}_{G}^{*}(K, M)$ such that

$$
\mathcal{I}_{G}^{*}(K, M)(G / G)=H_{G}^{*}(K, M) .
$$

This functor can be extended to a functor

$$
\mathcal{I}_{G}^{*}: G-\mathrm{CW}^{\mathrm{op}} \times\left(O_{G}^{\mathrm{op}}, \mathrm{Ab}\right) \times \mathbb{H}_{G}^{\mathrm{op}} \rightarrow \mathrm{Ab}^{*},
$$

where $(-,-)$ denotes the category of functors and $\mathrm{Ab}^{*}$ denotes the category of graded abelian groups. Hence we can regard $\mathcal{I}_{G}^{*}$ as a Hecke structure on Bredon cohomology. This structure was defined in [10]. It is induced by a functor

$$
\gamma: \mathbb{H}_{G}^{\mathrm{op}} \times\left(O_{G}^{\mathrm{op}}, \mathrm{Ab}\right) \rightarrow\left(O_{G}^{\mathrm{op}}, \mathrm{Ab}\right)
$$

such that $\gamma(G / G, M)=M$ for every coefficient system $M$. The structure $\mathcal{I}_{G}^{*}$, after restriction to $O_{G}^{\text {op }}$, is not equal to $\mathcal{H}_{G}^{*}$.

We also give another equivalent definition of a Hecke structure on Bredon cohomology. We introduce a functor $\delta: O_{G} \times G$-CW $\rightarrow G$-CW such that $\delta(G / G, K)=K$, for every $G$-CW-complex $K$. Then we show that

$$
H_{G}^{*}(K, \gamma(G / H, M)) \cong H_{G}^{*}(\delta(G / H, K), M),
$$

for every coefficient system $M$.

If $L$ is a local coefficient system on $K$, then we can define the coefficient system $\mathcal{H}_{G}^{*}(K, L)$ in such a way that

$$
\mathcal{H}_{G}^{*}(K, L)(G / H)=H_{G}^{*}\left(G / H \times K, L p_{G / H}\right)=H_{H}^{*}(K, L \mid H),
$$

where $p_{G / H}$ denotes the projection $G / H \times K \rightarrow K$. We show that this system can be extended to a Hecke functor and that

$$
H_{G}^{*}\left(G / H \times K, L p_{G / H}\right) \cong H_{G}^{*}\left(\delta(G / H, K), L \delta\left(\pi_{G / H}, \operatorname{id}_{K}\right)\right),
$$

where $\pi_{G / H}$ denotes the map $G / H \rightarrow G / G$. Hence, if we define

$$
\mathcal{I}_{G}^{*}(K, L)=H_{G}^{*}\left(\delta(G / H, K), L \delta\left(\pi_{G / H}\right)\right),
$$

then $\mathcal{H}_{G}^{*} \cong \mathcal{I}_{G}^{*}$ in this case.

We show that there is a map

$$
\phi(G / H, K): G / H \times K \rightarrow \delta(G / H, K)
$$

natural in $G / H$ and $K$ and such that $p_{G / H}=\delta\left(\pi_{G / H}\right) \phi(G / H, K)$. This map induces, for every coefficient system $M: O_{G}^{\text {op }} \rightarrow \mathrm{Ab}$, a natural trans- 
formation of coefficient systems

$$
\phi^{*}(K, M): \mathcal{I}_{G}^{*}(K, M) \rightarrow \mathcal{H}_{G}^{*}(K, M) .
$$

The natural transformation $\phi^{*}(-, M)$ of contravariant functors from $G$ $\mathrm{CW} \times O_{G}$ to the category of graded abelian groups is a natural equivalence if and only if $M$ is a constant functor. In the case where $M$ is a Hecke functor, $\phi^{*}(K, M)$ is a natural transformation of Hecke functors. Let $M \theta_{G}(K)$ be the local coefficient system on $K$ determined by $M$. Then $\phi^{*}(K, M)$ can be considered as the map induced by a natural transformation of local coefficient systems on $G / H \times K$

$$
\varphi\left(M, p_{G / H}\right): M \theta_{G}(K) p_{G / H} \rightarrow M \theta_{G}(G / H \times K) .
$$

One of the results of this paper is the construction of a spectral sequence $E_{*}^{p, q}$ such that

$$
E_{2}^{p, q}=H_{G}^{p}\left(K^{\prime}, \mathcal{I}_{G}^{q}(K, L)\right)
$$

where $K^{\prime}$ is a certain $G$-CW-complex. We discuss the cases where $E_{2}^{p, q} \Rightarrow$ $H_{G}^{p+q}(K, L)$.

Some of the results stated in Section 1 will be proved in Section 2. We show that the Hecke structure on Bredon cohomology can be described in well known terms of category theory. We also prove that this construction can be generalized to the case of functors from a category associated to a $G$-poset to Ab. We shall begin Section 2 with a definition of such a category.

The author wishes to thank the referee for his careful reading of the manuscript and his useful suggestions and observations.

1. Main results. We begin with the definition of Hecke structure on the category of $G$-coefficient systems. We need the following notation.

Let $\mathbb{Z}(G)$-Mod denote the category of left $\mathbb{Z}(G)$-modules. The category $\mathbb{H}_{G}$ can be considered as a full subcategory of $\mathbb{Z}(G)$-Mod, because there is a natural inclusion $\iota: \mathbb{H}_{G} \rightarrow \mathbb{Z}(G)$-Mod given by $\iota(G / H)=\mathbb{Z}(G / H)$. The natural inclusion $O_{G} \rightarrow \mathbb{H}_{G}$ will be denoted by i. For any $G$-map $f$ : $G / H \rightarrow G / H^{\prime}, \mathfrak{i}(f)$ is the $\mathbb{Z}(G)$-module homomorphism $\mathbb{Z}(f): \mathbb{Z}(G / H) \rightarrow$ $\mathbb{Z}\left(G / H^{\prime}\right)$.

We shall consider the functor $\beta: \mathbb{Z}(G)-\operatorname{Mod}^{\text {op }} \rightarrow\left(\mathbb{Z}(G)-\mathrm{Mod}^{\mathrm{op}}, \mathrm{Ab}\right)$ such that for any $\mathbb{Z}(G)$-modules $A$ and $A^{\prime}$

$$
\beta(A)\left(A^{\prime}\right)=\operatorname{Hom}_{\mathbb{Z}(G)}\left(A \otimes A^{\prime}, \mathbb{Z}\right)
$$

where $\mathbb{Z}=\mathbb{Z}(G / G)$ is the trivial $\mathbb{Z}(G)$-module. If $A=\mathbb{Z}$, then $\beta(\mathbb{Z})$ is the Yoneda functor $\operatorname{Hom}_{\mathbb{Z}(G)}(-, Z)$.

Let $\mathbb{C}$ and $\mathbb{C}^{\prime}$ be small categories. For any functors $\alpha: \mathbb{C} \rightarrow \mathbb{Z}(G)$-Mod and $\alpha^{\prime}: \mathbb{C}^{\prime} \rightarrow \mathbb{Z}(G)$-Mod the functor $\beta$ induces a functor

$$
\beta\left(\alpha, \alpha^{\prime}\right): \mathbb{C}^{\mathrm{op}} \rightarrow\left(\mathbb{C}^{\prime \mathrm{op}}, \mathrm{Ab}\right),
$$


which is the composition $\left(\alpha^{\prime}\right.$, id $) \beta \alpha$. If $\alpha$ is an additive functor, then so is $\beta\left(\alpha, \alpha^{\prime}\right)$. The functor $\beta(\iota, \iota \mathrm{i}): \mathbb{H}_{G}^{\mathrm{op}} \rightarrow\left(O_{G}^{\mathrm{op}}, \mathrm{Ab}\right)$ will be denoted by $\beta^{\prime}$. It follows from the definition that, for any subgroups $H$ and $H^{\prime}$ of $G$,

$$
\beta^{\prime}(G / H)\left(G / H^{\prime}\right)=\operatorname{Hom}_{\mathbb{Z}(G)}\left(\mathbb{Z}(G / H) \otimes \mathbb{Z}\left(G / H^{\prime}\right), \mathbb{Z}\right) .
$$

Hence $\beta^{\prime}(G / G)\left(G / H^{\prime}\right)=\mathbb{Z}$ and $\beta^{\prime}(G / G)(f)=\operatorname{id}_{\mathbb{Z}}$ whenever $H^{\prime}$ is a subgroup of $G$ and $f$ is a morphism of $O_{G}$.

Assume now that $\mathbb{C}$ is a small category. For any functors $M, M^{\prime}: \mathbb{C} \rightarrow$ $\mathrm{Ab}$, we shall denote by $M \otimes M^{\prime}$ the functor from $\mathbb{C}$ to Ab such that $(M \otimes$ $\left.M^{\prime}\right)(-)=M(-) \otimes M^{\prime}(-)$. Let $\mathbb{Z}_{\mathbb{C}}: \mathbb{C} \rightarrow$ Ab denote the constant functor such that, for every object $c$ of $\mathbb{C}, \mathbb{Z}_{\mathbb{C}}(c)=\mathbb{Z}$, and for every morphism $m$ of $\mathbb{C}, \mathbb{Z}_{\mathbb{C}}(m)=\operatorname{id}_{\mathbb{Z}}$. Then $M \otimes \mathbb{Z}_{\mathbb{C}}=\mathbb{Z}_{\mathbb{C}} \otimes M=M$.

Assume that $\alpha$ is a functor from $\mathbb{C}$ to $\mathbb{Z}(G)$-Mod. Let

$$
\alpha^{\prime \prime}: \mathbb{Z}(G)-\operatorname{Mod}^{\mathrm{op}} \times\left(\mathbb{C}^{\mathrm{op}}, \mathrm{Ab}\right) \rightarrow\left(\mathbb{C}^{\mathrm{op}}, \mathrm{Ab}\right)
$$

be the functor such that, for any $\mathbb{Z}(G)$-module $A, \alpha^{\prime \prime}(A, M)=\beta(\mathrm{id}, \alpha)(A)$ $\otimes M$.

1.1. Proposition. The functor $\alpha^{\prime \prime}$ has the following properties:

(i) $\alpha^{\prime \prime}(A, M)(c)=\operatorname{Hom}_{\mathbb{Z}(G)}(A \otimes \alpha(c), \mathbb{Z}) \otimes M(c)$ whenever $c$ is an object of $\mathbb{C}$.

(ii) $\alpha^{\prime \prime}(A, M)=\alpha^{\prime \prime}\left(A, \mathbb{Z}_{\mathbb{C}_{\text {op }}}\right) \otimes M$. $=M$.

(iii) If $\alpha=\iota \mathrm{i} \alpha_{0}$ where $\alpha_{0}$ is a functor from $\mathbb{C}$ to $O_{G}$ then $\alpha^{\prime \prime}(\mathbb{Z}(G / G), M)$

Proof. (i) and (ii) follow immediately from the definition. (iii) holds because $(\iota \mathrm{i})^{\prime \prime}\left(\mathbb{Z}(G / G), \mathbb{Z}_{O_{G}^{\text {op }}}\right)=\operatorname{Hom}_{\mathbb{Z}(G)}(\mathbb{Z}(-), \mathbb{Z})=\mathbb{Z}_{O_{G}^{\text {op }}}$.

In particular, for $\mathbb{C}=O_{G}$ we obtain the following fact.

1.2. Corollary. Let the functor $\gamma: \mathbb{H}_{G}^{\mathrm{op}} \times\left(O_{G}^{\mathrm{op}}, \mathrm{Ab}\right) \rightarrow\left(O_{G}^{\mathrm{op}}, \mathrm{Ab}\right)$ be the composition $(\iota \mathrm{i})^{\prime \prime}(\iota, \mathrm{id})$. Then, for every coefficient system $M, \gamma(-, M)=$ $\beta^{\prime}(-) \otimes M$ and, in particular, $\gamma(G / G, M)=M$.

We shall also use the notation

$$
\gamma(-, M)=M[-] \quad \text { and } \quad \mathbb{Z}_{O_{G}^{\text {op }}}=\mathbb{Z}_{O_{G}} .
$$

Hence, for every coefficient system $M, M[G / H]=\mathbb{Z}_{O_{G}}[G / H] \otimes M$ and $M[G / G]=M$. If $H^{\prime}$ is a subgroup of $G$, then

$$
M[G / H]\left(G / H^{\prime}\right)=\operatorname{Hom}_{\mathbb{Z}(G)}\left(\mathbb{Z}(G / H) \otimes \mathbb{Z}\left(G / H^{\prime}\right), \mathbb{Z}\right) \otimes M\left(G / H^{\prime}\right) .
$$

1.3. Definition. We define a Hecke structure on Bredon cohomology as a functor

$$
\mathcal{I}_{G}^{*}(-,-)(-): G-\mathrm{CW}^{\mathrm{op}} \times\left(O_{G}^{\mathrm{op}}, \mathrm{Ab}\right) \times \mathbb{H}_{G}^{\mathrm{op}} \rightarrow \mathrm{Ab}^{*}
$$

given by $\mathcal{I}_{G}^{*}(K, M)(-)=H_{G}^{*}(K, M[-])$. 
We can also define a Hecke structure on Bredon cohomology with local coefficients. Assume that $K$ is a $G$-CW-complex. This means that $K$ is a CW-complex and that $G$ acts on $K$ in such a way that for every subgroup $H$ of $G$ the fixed point set $K^{H}$ is a subcomplex of $K$. Let $\mathcal{K}$ be the category defined in [2], Ch. I.5. Its objects are the finite subcomplexes of $K$ and its morphisms are the compositions of the inclusions and the maps induced by the operation by the elements of $G$. We shall also use the notation $\mathcal{K}_{G}=\mathcal{K}$. Assume that $f: K_{0} \rightarrow K$ is a $G$-CW-map. Then the map $\mathrm{f}: \mathcal{K}_{0} \rightarrow \mathcal{K}$ is defined in such a way that, for each subcomplex $K_{1}$ of $K_{0}, f\left(K_{1}\right)$ is the smallest subcomplex $K\left(f\left(K_{1}\right)\right)$ of $K$ which contains $f\left(K_{1}\right)$.

The local coefficient systems on $K$ in the sense of Bredon are the covariant functors on $\mathcal{K}$. If $L$ is a local coefficient system on $K$, then the local coefficient system $L f$ will also be denoted by $L f$. Let $\mathcal{L}_{G}$ be the category whose objects are the pairs $(K, L)$ where $K$ is a $G$-CW-complex and $L$ is a functor from $\mathcal{K}_{G}$ to $\mathrm{Ab}$. The morphisms of $\mathcal{L}_{G}$ are the pairs $(f, \varrho)$ : $\left(K_{0}, L_{0}\right) \rightarrow(K, L)$ where $f: K_{0} \rightarrow K$ is a $G$-CW-map and $\varrho: L f \rightarrow L_{0}$ is a natural transformation of functors. In [2], Ch. I.6, 7, Bredon defined the cohomology functor $H_{G}^{*}$ from the category $\mathcal{L}_{G}^{\text {op }}$ to the category $\mathrm{Ab}^{*}$ of graded abelian groups. In this paper we shall use a slightly modified definition of the term "local coefficient system".

Let $\mathcal{K}(G)$ be the full subcategory of $\mathcal{K}_{G}$ whose objects are all subcomplexes of $K$ of the form $K(s)$, where $s$ is a cell of $K$, and $K(s)$ is the smallest subcomplex of $K$ containing $s$. We shall consider the functors from the category $\mathcal{K}(G)$ to the category $\mathrm{Ab}$ and call them the local coefficient systems on $K$. Assume that $L: \mathcal{K}(G) \rightarrow$ Ab. We shall also use the notation $L(K(s))=L(s)$. Let $\mathrm{u}: \mathcal{K}(G) \rightarrow \mathcal{K}_{G}$ be the natural inclusion of categories. We shall denote by $L^{e}: \mathcal{K}_{G} \rightarrow$ Ab the left Kan extension of the functor $L$. It follows from the definition that, for every subcomplex $K^{\prime}$ of $K$,

$$
L^{e}\left(K^{\prime}\right)=\operatorname{colim}_{\mathcal{K}_{G} / K^{\prime}} L / K^{\prime} .
$$

Here $\mathcal{K}_{G} / K^{\prime}$ is the full subcategory of $\mathcal{K}_{G}$ whose objects are all subcomplexes of the form $K(s)$ contained in $K^{\prime}$ and $L / K^{\prime}$ is the restriction of $L$. There exists a canonical functor $e: \mathcal{L}_{G} \rightarrow \mathcal{L}_{G}$ such that, for every pair $(K, L), \mathrm{e}(K, L)=\left(K,(L \mathrm{u})^{e}\right)$ and a canonical natural transformation of functors $\mathrm{t}: \mathrm{id}_{\mathcal{L}_{G}} \rightarrow \mathrm{e}$ such that, for every pair $(K, L), \mathrm{t}(K, L)=\left(\operatorname{id}_{K}, \mathrm{t}_{L}\right)$, and the homomorphism

$$
H_{G}^{*}\left(K, \mathrm{t}_{L}\right): H_{G}^{*}\left(K,(L \mathrm{u})^{e}\right) \rightarrow H_{G}^{*}(K, L)
$$

is the identity map. Let $\mathcal{L}(G)$ denote the category of all pairs $(K, L)$ such that $L: \mathcal{K}(G) \rightarrow$ Ab. The morphisms of $\mathcal{L}(G)$ are the pairs $(f, \varrho)$ : $\left(K_{0}, L_{0}\right) \rightarrow(K, L)$ where $f: K_{0} \rightarrow K$ is a $G$-CW-map and $\varrho:\left(L^{e} f\right) \mathrm{u} \rightarrow L_{0}$ is a natural transformation of functors. We shall also use the notation 
$\left(L^{e} f\right) \mathrm{u}=L f$. It follows from the above considerations that we can define the functor $H_{G}^{*}$ from $\mathcal{L}(G)$ to $\mathrm{Ab}^{*}$ in such a way that, for every pair $(K, L)$, $H_{G}^{*}(K, L)=H_{G}^{*}\left(K, L^{e}\right)$.

Assume that $f: K_{0} \rightarrow K$ is a $G$-CW-map satisfying the condition that, for every cell $s_{0}$ of $K_{0}$, there exists a cell $s$ of $K$ such that $K\left(f\left(K_{0}\left(s_{0}\right)\right)\right)=$ $K(s)$. Then $f$ induces a functor $f_{G}: \mathcal{K}_{0}(G) \rightarrow K(G)$ and, for every local coefficient system $L$ on $K$, we have $L f=L f_{G}$. One can easily check that in this case, for any functor $L_{1}: \mathcal{K}_{G} \rightarrow \mathrm{Ab},\left(L_{1} f\right) \mathrm{u}=\left(L_{1} \mathrm{u}\right) f$.

Let $\theta: \mathcal{K}^{\mathrm{op}} \rightarrow O_{G}$ be the canonical contravariant functor defined by Bredon in [2], Ch. I.5. We shall use its restriction $\theta_{G}(K): \mathcal{K}(G) \rightarrow O_{G}$. For every cell $s$ of $K, \theta_{G}(K)(K(s))=G / G_{s}$ where $G_{s}=\{g \in G: g s=s\}$.

1.4. Corollary. There exists a functor $\gamma_{K}: \mathbb{H}_{G}^{\mathrm{op}} \times(\mathcal{K}(G), \mathrm{Ab}) \rightarrow$ $(\mathcal{K}(G), \mathrm{Ab})$ such that

$\gamma_{K}(G / H, L)(K(s))=\operatorname{Hom}_{\mathbb{Z}(G)}\left(\mathbb{Z}(G / H) \otimes \mathbb{Z}\left(G / G_{s}\right), \mathbb{Z}\right) \otimes L(K(s))$.

Proof. This follows from 1.1 for $\gamma_{K}=\left(\iota \mathrm{i} \theta_{G}\right)^{\prime \prime}(\iota, \mathrm{id})$.

We shall also use the notation $L[G / H]=\gamma_{K}(G / H, L)$.

1.5. Definition. We define a Hecke structure on Bredon cohomology of $K$ with local coefficients as a functor

$$
\mathcal{I}_{G}^{*}(K,-)(-):(\mathcal{K}(G), \mathrm{Ab}) \times \mathbb{H}_{G}^{\text {op }} \rightarrow \mathrm{Ab}^{*}
$$

given by $\mathcal{I}_{G}^{*}(K, L)(G / H)=H_{G}^{*}(K, L[G / H])$.

One can also prove that there exists a functor $\gamma: \mathbb{H}_{G}^{\text {op }} \times \mathcal{L}_{G} \rightarrow \mathcal{L}_{G}$ such that, for every $G$-CW-complex $K, \gamma(-,(K,-))=\gamma_{K}$. Thus we can consider the functor

$$
\mathcal{I}_{G}^{*}(-,-)(-): \mathcal{L}_{G}^{\mathrm{op}} \times \mathbb{H}_{G}^{\mathrm{op}} \rightarrow \mathrm{Ab}^{*}
$$

In order to avoid complications, we restrict ourselves to the case of the local coefficient systems defined on a fixed $G$-CW-complex $K$.

Let $\vartheta: \mathbb{Z}(G)$-Mod $\rightarrow\left(\mathbb{H}_{G}^{\text {op }}, \mathrm{Ab}\right)$ be the functor induced by the Yoneda functor $\varphi: \mathbb{Z}(G)$-Mod $\rightarrow\left(\mathbb{Z}(G)-\operatorname{Mod}^{\mathrm{op}}, \mathrm{Ab}\right)$. This means that, for every $\mathbb{Z}(G)$-module $N$ and every subgroup $H$ of $G$,

$$
\vartheta(N)(G / H)=\operatorname{Hom}_{\mathbb{Z}(G)}(Z(G / H), N),
$$

and for every $\mathbb{Z}(G)$-homomorphism $f: \mathbb{Z}\left(G / H^{\prime}\right) \rightarrow \mathbb{Z}(G / H)$,

$$
\vartheta(N)(f)=\operatorname{Hom}_{\mathbb{Z}(G)}\left(f, \operatorname{id}_{N}\right) .
$$

For any $\mathbb{Z}(G)$-module cochain complex $C^{*}$, the functor $\vartheta$ induces a Hecke functor cochain complex $c^{*}=\vartheta\left(C^{*}\right)$ such that, for every natural number $n$,

$$
c^{n}(G / H)=\vartheta\left(C^{n}\right)(G / H)=\left(C^{n}\right)^{H} .
$$


The functor $\vartheta$ also gives us, for each natural number $n$, the Hecke functor $h^{n}$, the $n$th cohomology functor of the cochain complex $c^{*}$. Let $\mathbb{Z}(G)-\operatorname{Mod}^{\mathrm{c}}$ denote the category of $\mathbb{Z}(G)$-module cochain complexes. We define a functor

$$
\mathrm{h}: \mathbb{Z}(G)-\operatorname{Mod}^{\mathrm{c}} \times \mathbb{H}_{G}^{\mathrm{op}} \rightarrow \mathrm{Ab}^{*}
$$

in such a way that, for every $\mathbb{Z}(G)$-module cochain complex $C^{*}, \mathrm{~h}\left(C^{*},-\right)=$ $h^{*}$.

Let $K$ be a $G$-CW-complex. Bredon in [2] introduced a functor

$$
C^{*}(K,-):\left(\mathcal{K}_{G}, \mathrm{Ab}\right) \rightarrow \mathbb{Z}(G)-\operatorname{Mod}^{\mathrm{c}},
$$

such that, for every functor $L: \mathcal{K}_{G} \rightarrow \mathrm{Ab}$,

$$
H_{G}^{*}(K, L)=\mathrm{h}\left(C^{*}(K, L), G / G\right) .
$$

Assume now that $L: \mathcal{K}(G) \rightarrow$ Ab. Then we define

$$
C^{*}(K, L)=C^{*}\left(K, L^{e}\right) .
$$

Let $S_{n} K$ denote the $G$-set of all $n$-cells of $K$. Then

$$
C^{n}(K, L)=\prod_{s \in S_{n} K} L(s) .
$$

If $g \in G$ and $\ell=(\ell(s)) \in C^{n}(K, L)$, then $g \ell=((g \ell)(s))$, where

$$
(g \ell)(s)=L(g) \ell\left(g^{-1} s\right)
$$

and $L(g)$ denotes the map $L\left(g: K\left(g^{-1} s\right) \rightarrow K(s)\right)$. Assume that $\alpha: L \rightarrow L^{\prime}$ is a natural transformation of coefficient systems on $K$. Then the group homomorphism $H_{G}^{n}(K, \alpha)$ is induced by the map $\prod_{s \in S_{n} K} \alpha(s)$. Thus we obtain a functor

$$
C^{*}(K,-):(\mathcal{K}(G), \mathrm{Ab}) \rightarrow \mathbb{Z}(G)-\operatorname{Mod}^{\mathrm{c}} .
$$

In Section 2 we prove the following result.

1.6. Proposition. There exists a natural isomorphism of functors from $(\mathcal{K}(G), \mathrm{Ab}) \times \mathbb{H}_{G}^{\mathrm{op}}$ to $\mathrm{Ab}^{*}$

$$
\mathcal{I}_{G}^{*}(K,-)(-) \rightarrow \mathrm{h}\left(C^{*}(K,-),-\right) .
$$

Assume now that $M: O_{G}^{\text {op }} \rightarrow \mathrm{Ab}$ is a coefficient system for the group $G$. Then by the definition $([2])$

$$
C^{*}(K, M)=C^{*}\left(K, M \theta_{G}(K)\right), \quad H_{G}^{*}(K, M)=H_{G}^{*}\left(K, M \theta_{G}(K)\right) .
$$

If $\beta: M \rightarrow M^{\prime}$ is a natural transformation of generic coefficient systems then the map $C^{*}(K, \beta): C^{*}(K, M) \rightarrow C^{*}\left(K, M^{\prime}\right)$ is given by the products of maps $\prod_{s \in S_{n} K} \beta\left(G / G_{s}\right)$. This map induces the graded group homomorphism $H^{*}(K, \beta)$.

The following result is an immediate consequence of 1.6. 
1.7. Corollary. There exists a natural isomorphism of functors from $G-\mathrm{CW}^{\mathrm{op}} \times\left(O_{G}^{\mathrm{op}}, \mathrm{Ab}\right) \times \mathbb{H}_{G}^{\mathrm{op}}$ to $\mathrm{Ab}^{*}$

$$
\mathcal{I}_{G}^{*}(-,-)(-) \rightarrow \mathrm{h}\left(C^{*}(-,-),-\right) .
$$

Let $f: K_{0} \rightarrow K$ be a $G$-CW-map. For any local coefficient system $L: \mathcal{K}(G) \rightarrow \mathrm{Ab}$, we shall denote by $H_{G}^{*}(f, L)$ the group homomorphism $H_{G}^{*}(K, L) \rightarrow H_{G}^{*}\left(K_{0}, L f\right)$ which is determined by the map $\left(f, \operatorname{id}_{L f}\right)$. The homomorphism $H_{G}^{*}(f, L)$ is induced by the $\mathbb{Z}(G)$-module cochain complex homomorphism $C^{*}(f, L): C^{*}(K, L) \rightarrow C^{*}\left(K_{0}, L f\right)$, which can be described in the following way. Let $C_{*}(K, \mathbb{Z})$ denote the cellular chain complex of $K$ and let $C_{*}(f, \mathbb{Z}): C_{*}\left(K_{0}, \mathbb{Z}\right) \rightarrow C_{*}(K, \mathbb{Z})$ be the chain map induced by $f$. Assume that $s_{0} \in S_{n} K_{0}$. If $C_{*}(f, \mathbb{Z})\left(s_{0}\right)=\sum_{i=1}^{r} n_{i} s_{i}$ where $s_{i} \in S_{n} K$ for $i=1, \ldots, r$, then, for any $\ell \in C^{n}(K, L)$,

$$
\left(C^{n}(f, L) \ell\right)\left(s_{0}\right)=\sum_{i=1}^{r} n_{i} \lambda_{i} \ell\left(s_{i}\right)
$$

where, for $i=1, \ldots, r, \lambda_{i}$ is the structural map

$$
L\left(s_{i}\right) \rightarrow \operatorname{colim}_{K(s) \subseteq K\left(f\left(K_{0}\left(s_{0}\right)\right)\right)} L(s)=L f\left(s_{0}\right) .
$$

Assume now that $M$ is a generic $G$-coefficient system. Then $f$ induces a homomorphism

$$
H_{G}^{*}(f, M): H_{G}^{*}(K, M) \rightarrow H_{G}^{*}\left(K_{0}, M\right)
$$

which is determined by appropriate $\mathbb{Z}(G)$-module homomorphisms

$$
C^{n}(f, M): C^{n}(K, M) \rightarrow C^{n}\left(K_{0}, M\right)
$$

such that, for $s_{0} \in S_{n} K_{0}$ and $m \in C^{n}(K, M)$,

$$
C^{n}(f, M)(m)\left(s_{0}\right)=\sum_{i=1}^{r} n_{i} \mu_{i} m\left(s_{i}\right)
$$

where, for $i=1, \ldots, r, \mu_{i}: M\left(G / G_{s_{i}}\right) \rightarrow M\left(G / G_{s_{0}}\right)$ is the map induced by the inclusion $G_{s_{0}} \subseteq G_{s_{i}}$.

We also have a natural transformation of local coefficient systems on $K_{0}$

$$
\varphi(M, f): M \theta_{G}(K) f \rightarrow M \theta_{G}\left(K_{0}\right)
$$

such that, for every cell $s_{0}$ of $K_{0}$, the map

$$
\varphi(M, f)\left(s_{0}\right): \operatorname{colim}_{K(s) \subseteq K\left(f\left(K_{0}\left(s_{0}\right)\right)\right)} M\left(G / G_{s}\right) \rightarrow M\left(G / G_{s_{0}}\right)
$$

is induced by the inclusions $G_{s_{0}} \subseteq G_{s}$. It is easy to check that

$$
H_{G}^{*}\left(K_{0}, \varphi(M, f)\right) H_{G}^{*}\left(f, M \theta_{G}(K)\right)=H_{G}^{*}(f, M) .
$$


For a $G$-map $f: \widetilde{K} \rightarrow K$ we shall denote by $P(f)$ the fiber product over $K / G$ of $f / G: \widetilde{K} / G \rightarrow K / G$ and the projection to the orbit space $\pi(K): K \rightarrow K / G$. We shall consider $P(f)$ as a subset of $(\widetilde{K} / G) \times K$. The group $G$ acts on $P(f)$ by the action on the second coordinate. There are two structural maps $f_{1}: P(f) \rightarrow K$ and $f_{2}: P(f) \rightarrow \widetilde{K} / G$ such that $\pi(K) f_{1}=(f / G) f_{2}$. Let $\phi: \widetilde{K} \rightarrow P(f)$ be the unique map such that $f=f_{1} \phi$ and $\pi(\widetilde{K})=f_{2} \phi$. Then $\phi / G$ is a homeomorphism and $\left(f_{2} \phi\right) / G$ is the identity map on $\widetilde{K} / G$. Further, $G_{x}=G_{f_{1}(x)}$ for any $x$ in $P(f)$. It is clear that $P(f)=\widetilde{K}$ if $G_{k}=G_{f(k)}$ for each $k$ in $\widetilde{K}$. Assume that $f=f^{\prime} f^{\prime \prime}$, where $f^{\prime}: K^{\prime \prime} \rightarrow K$ and $f^{\prime \prime}: \widetilde{K} \rightarrow K^{\prime \prime}$ are $G$-maps. Then there exist unique $G$-maps $p_{f^{\prime \prime}}: P(f) \rightarrow P\left(f^{\prime}\right)$ and $\pi_{f^{\prime}}: P\left(f^{\prime \prime}\right) \rightarrow P(f)$ such that the diagrams

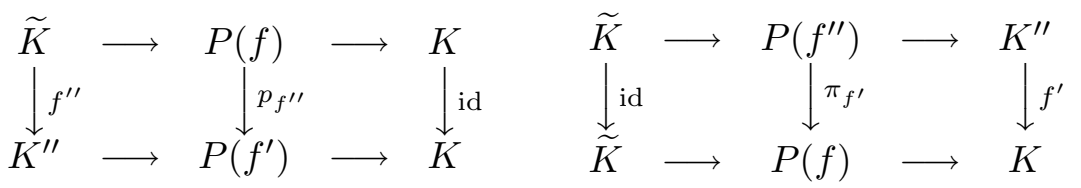

commute. If $f$ is a $G$-CW-map, then the topology of $P(f)$ is induced from the topology of the product $(\widetilde{K} / G) \times K$ in the category of $k$-spaces.

Assume now that $\widetilde{K}$ is the product $K^{\prime} \times K$ of two $G$-sets with diagonal action of $G$ and that $f=p_{K^{\prime}}: K^{\prime} \times K \rightarrow K$ is the projection onto the second coordinate. In this case we shall use the notation $P_{G}\left(K^{\prime}, K\right)=P\left(p_{K^{\prime}}\right)$. If $K^{\prime}$ is a $G$-CW-complex, then $K^{\prime} \times K$ is a product in the category of $k$ spaces. Since open cells of $K^{\prime} \times K$ are products of open cells of $K^{\prime}$ and $K$, $P_{G}\left(K^{\prime}, K\right)$ has the natural structure of a $G$-CW-complex whose open cells are subspaces of the form $\left\{\left(\left(g k^{\prime}, g k\right), k\right): k \in s, k^{\prime} \in s^{\prime}\right\}$ where $g$ is a fixed element of $G, s$ is an open cell of $K$ and $s^{\prime}$ is an open cell of $K^{\prime}$. We shall denote by $S(K)$ the $G$-set of all open cells of $K$. Thus

$$
S\left(P_{G}\left(K^{\prime}, K\right)\right)=P_{G}\left(S\left(K^{\prime}\right), S(K)\right) .
$$

We can extend, in a natural way, the construction above to a functor

$$
P_{G}: G-\mathrm{CW} \times G-\mathrm{CW} \rightarrow G-\mathrm{CW} .
$$

Let $P: G$-CW $\times G$-CW $\rightarrow G$-CW be the product functor; i.e., $P\left(K^{\prime}, K\right)=$ $K^{\prime} \times K$ together with the diagonal action of $G$. There exists a natural transformation of functors $\phi: P \rightarrow P_{G}$ such that $\phi\left(K^{\prime}, K\right)$ is the map determined by the projection $p_{K^{\prime}}: K^{\prime} \times K \rightarrow K$ and by the projection $K^{\prime} \times K \rightarrow\left(K^{\prime} \times K\right) / G$ to the orbit space. This natural transformation has the following properties.

1.8. Proposition. (i) Let $L$ be a local coefficient system on $K$. Then, for every $G$-CW-complex $K^{\prime}$, the $G$-cellular map $\phi\left(K^{\prime}, K\right)$ induces an iso- 
morphism of cohomology groups

$$
H_{G}^{*}\left(P_{G}\left(K^{\prime}, K\right), L\left(p_{K^{\prime}}\right)_{1}\right) \rightarrow H_{G}^{*}\left(K^{\prime} \times K, L p_{K^{\prime}}\right) .
$$

(ii) Let $M: O_{G}^{\mathrm{op}} \rightarrow \mathrm{Ab}$ be a generic $G$-coefficient system. Then

$$
M \theta_{G}(K)\left(p_{K^{\prime}}\right)_{1}=M \theta_{G}\left(P_{G}\left(K^{\prime}, K\right)\right)
$$

and the map $\phi\left(K^{\prime}, K\right)$ induces an isomorphism

$$
H_{G}^{*}\left(P_{G}\left(K^{\prime}, K\right), M\right) \rightarrow H_{G}^{*}\left(K^{\prime} \times K, M \theta_{G}(K) p_{K^{\prime}}\right) .
$$

Proof. (i) Set $L_{1}=L\left(p_{K^{\prime}}\right)_{1}$. It is clear that $L p_{K^{\prime}}=L_{1} \phi\left(K^{\prime}, K\right)$. Hence it is sufficient to prove that the maps $C^{n}\left(\phi\left(K^{\prime}, K\right), L_{1}\right)^{G}$ are isomorphisms and this follows from the fact that $\phi\left(K^{\prime}, K\right) / G$ is a homeomorphism such that $\left(\left(p_{K^{\prime}}\right)_{2} \phi\left(K^{\prime}, K\right)\right) / G=\operatorname{id}_{\left(K^{\prime} \times K\right) / G}$.

(ii) This assertion is a consequence of the fact that, for every $x$ in $P_{G}\left(K^{\prime}, K\right), G_{x}=G_{k}$ where $k=\left(p_{K^{\prime}}\right)_{1} x$

The category $O_{G}$ can be considered as a full subcategory of the category $G$-CW. The restriction of the functor $P_{G}$ to the category $O_{G} \times G$-CW will be denoted by $\delta$. It is obvious that $\delta(G / G, K)=K$ and that $\left(p_{G / H}\right)_{1}$ is equal to $\delta\left(\pi_{G / H}, \operatorname{id}_{K}\right)$ where $\pi_{G / H}: G / H \rightarrow G / G$.

We shall use the notation

$$
\begin{aligned}
& H_{G}^{*}(\delta(G / H, K), L)=H_{G}^{*}\left(\delta(G / H, K), L \delta\left(\pi_{G / H}, \operatorname{id}_{K}\right)\right), \\
& C_{G}^{*}(\delta(G / H, K), L)=C_{G}^{*}\left(\delta(G / H, K), L \delta\left(\pi_{G / H}, \operatorname{id}_{K}\right)\right) .
\end{aligned}
$$

Let $u(G / H, K): K \rightarrow \delta(G / H, K)$ be the map determined by the identity $\operatorname{id}_{K}: K \rightarrow K$ and by the map $u_{0}: K \rightarrow(G / H \times K) / G$ such that, for every $k \in K, u_{0}(k)=[(e H, k)]$. It is clear that $u(G / H, K)$ is an $H$-CW-map and that, for every $G$-map $f: G / H \rightarrow G / H^{\prime}$ such that $f(e H)=e H^{\prime}$,

$$
\delta\left(f, \operatorname{id}_{K}\right) u(G / H, K)=u\left(G / H^{\prime}, K\right) .
$$

The map $u(G / H, K)$ induces, for every local coefficient system $L: \mathcal{K}(G) \rightarrow$ $\mathrm{Ab}$, a $\mathbb{Z}(H)$-module cochain complex homomorphism

$$
u^{*}(L, G / H): C^{*}(\delta(G / H, K), L) \rightarrow C^{*}(K, L) .
$$

Let $w^{*}(L, G / H): C^{*}(\delta(G / H, K), L)^{G} \rightarrow C^{*}(K, L)^{H}$ denote the restriction of $u^{*}(L, G / H)^{H}$ to the $\mathbb{Z}$-module $C^{*}(\delta(G / H, K), L)^{G}$.

1.9. Proposition. There exists a natural equivalence of functors from $(\mathcal{K}(G), \mathrm{Ab}) \times O_{G}^{\mathrm{op}}$ to $\mathrm{Ab}^{*}$

$$
\sigma^{*}: H_{G}^{*}(\delta(-, K),-) \rightarrow \mathrm{h}\left(C^{*}(K,-),-\right),
$$

such that, for each subgroup $H$ of $G$ and each local coefficient system $L$ on $K, \sigma^{*}(L, G / H)$ is induced by the cochain complex homomorphism $w^{*}(L, G / H)$.

In the proof of this proposition we shall use the following facts. 
Assume that $K$ is a $G$-CW-complex and that $H$ is a normal subgroup of $G$. Then $K / H$ has a $G$-CW-complex structure induced from $K$ and the projection to the orbit space $\omega: K \rightarrow K / H$ is a $G$-CW-map.

1.10. Lemma. Let $L$ be a local coefficient system on $K / H$. Then the image of the $\mathbb{Z}(G)$-module cochain complex monomorphism $C^{*}(K / H, L) \rightarrow$ $C^{*}(K, L \omega)$ is equal to $C^{*}(K, L \omega)^{H}$.

Proof. This follows immediately from the definitions of the cochain complex of Bredon cohomology and of the homomorphism induced by a $G$-CW-map.

Assume now that $K$ is a $G$-CW-complex. Let $K^{\prime \prime}=K \times_{K / G} K$ be the fiber product over $K / G$ of two projections $\pi: K \rightarrow K / G$ onto the orbit space. The group $G$ acts on $K^{\prime \prime}$ by the action on $K \times K$ and $K^{\prime \prime}$ has a natural structure of a $G \times G$-CW-complex. The set of cells of $K \times_{K / G} K$ is $S(K) \times S(K) / G S(K)$, where $S(K)$ is the cell set of $K$. Thus, we can assume that the cells of $K^{\prime \prime}$ are indexed by the set $\{(s, g s): s \in S(K), g \in G\}$. Let $p$ and $p^{\prime}$ be the structural maps from $K^{\prime \prime}$ to $K$. We can consider them as the $G \times G$-CW-projections $p^{\prime}: K^{\prime \prime} \rightarrow K^{\prime \prime} / G \times(e), p: K^{\prime \prime} \rightarrow K^{\prime \prime} /(e) \times G$.

1.11. Lemma. Assume that $L$ is a $G$-local coefficient system on $K$. Then there exists an isomorphism $\mathfrak{j}: L p \rightarrow L p^{\prime}$ of $G \times G$-local coefficient systems on $K^{\prime \prime}=K \times_{K / G} K$ such that, for every cell $s$ of $K, \mathfrak{j}(s, s)$ is the identity map.

Proof. Let $\left(s, s^{\prime}\right)$ be a cell of $K^{\prime \prime}$. Then there is $g$ in $G$ such that $s^{\prime}=g s$. We define $\mathrm{j}(s, g s): L(s) \rightarrow L(g s)$ to be $L([g])$, where $[g]: K(s) \rightarrow K(g s)$ is multiplication by $g$.

Proof of Proposition 1.9. Let $K$ be a $G$-CW-complex and let $H$ be a subgroup of $G$. We shall use the fact that there exists an isomorphism

$$
\mathrm{a}:(G / H \times K) / G \rightarrow K / H
$$

such that $\mathrm{a}(G(g H, k))=H g^{-1} k$ whenever $g \in G$ and $k \in K$. This implies that $K^{\prime \prime}=K \times_{K / G} K$, with the group action restricted to $(e) \times G$, is equal to $\delta(G /(e), K)$ and that $\delta(G / H, K)$ is equal to $K^{\prime \prime} /(H \times(e))$ as $(e) \times G$ $\mathrm{CW}$-complexes.

Let $\pi_{G / H}: G / H \rightarrow G / G$ be the natural projection. Then $\delta\left(\pi_{G /(e)}, \operatorname{id}_{K}\right)$ $=p^{\prime}: K^{\prime \prime} \rightarrow K^{\prime \prime} / G \times(e)$. Assume that $d_{G / H}: G /(e) \rightarrow G / H$ is the projection such that $d_{G / H}(g)=g H$ for $g \in G$. Then $\delta\left(d_{G / H}, \operatorname{id}_{K}\right)$ is the projection onto the orbit space of $H \times(e)$.

Let $L$ be a local coefficient system on $K$. From Lemmas 1.10 and 1.11 we conclude that there exist $\mathbb{Z}(G)=\mathbb{Z}((e) \times G)$-isomorphisms of cochain complexes

$$
C^{*}(\delta(G / H, K), L) \cong C^{*}\left(K^{\prime \prime}, L p^{\prime}\right)^{H \times(e)} \cong C^{*}\left(K^{\prime \prime}, L p\right)^{H \times(e)} .
$$


Lemma 1.10 also yields a $\mathbb{Z}(G \times(e))$-module isomorphism $C^{*}(K, L) \cong$ $C^{*}\left(K^{\prime \prime}, L p\right)^{(e) \times G}$. Hence we obtain isomorphisms

$$
C^{*}(\delta(G / H, K), L)^{G} \cong C^{*}\left(K \times_{K / G} K, L p\right)^{H \times G} \cong C^{*}(K, L)^{H} .
$$

Let $u=u(G / e, K)$. Since $p u=p^{\prime} u=\mathrm{id}_{K}$ and $\mathrm{j} u: L p u \rightarrow L p^{\prime} u$ is the identity map it follows that the isomorphisms above are induced by the $\operatorname{maps} w^{*}(L, G / H)$.

The $G$-complex $\delta(G / H, K)$ will also be denoted by $K[G / H]$. Thus $K[-]$ can be considered as a functor from $O_{G}$ to $G$-CW. If, for each $k$ in $K, G_{k}$ is a subgroup of $H$, then $\phi(G / H, K): G / H \times K \rightarrow K[G / H]$ is a $G$-CWhomeomorphism.

As immediate consequences of 1.8 and 1.9 we obtain the following results.

1.12. COROLlary. There exists a natural equivalence of functors from $(\mathcal{K}(G), \mathrm{Ab}) \times O_{G}^{\text {op }}$ to $\mathrm{Ab}^{*}$

$$
H_{G}^{*}(-\times K,-) \rightarrow \mathrm{h}\left(C^{*}(K,-),-\right),
$$

where, for every local coefficient system $L$ and every subgroup $H$ of $G$,

$$
H_{G}^{*}(G / H \times K, L)=H_{G}^{*}\left(G / H \times K, L p_{G / H}\right) .
$$

1.13. COROLlaRY. There exists a natural equivalence of functors from $G-\mathrm{CW}^{\mathrm{op}} \times\left(O_{G}^{\mathrm{op}}, \mathrm{Ab}\right) \times O_{G}^{\mathrm{op}}$ to $\mathrm{Ab}^{*}$

$$
H_{G}^{*}(-[-],-) \rightarrow \mathrm{h}\left(C^{*}(-,-),-\right) .
$$

1.6 and 1.7 yield the next results.

1.14. Corollary. Let $K$ be a $G$-CW-complex and let $H$ be a subgroup of $G$.

(i) Assume that $L$ is a local coefficient system on $K$. Then there are isomorphisms

$$
H_{G}^{*}(K[G / H], L) \cong H_{G}^{*}(G / H \times K, L) \cong H_{G}^{*}(K, L[G / H])
$$

which are natural with respect to $L$ in $(\mathcal{K}(G), \mathrm{Ab})$ and $G / H$ in $O_{G}$.

(ii) Let $M: O_{G}^{\mathrm{op}} \rightarrow \mathrm{Ab}$ be a coefficient system for the group $G$. Then there is an isomorphism

$$
z^{*}(K, M)(G / H): H_{G}^{*}(K[G / H], M) \rightarrow H_{G}^{*}(K, M[G / H])
$$

which is natural with respect to $K$ in $G$-CW, $G / H$ in $O_{G}$ and $M$ in $\left(O_{G}^{\mathrm{op}}, \mathrm{Ab}\right)$.

1.15. Corollary. (i) There exists a natural equivalence of functors $\mathcal{H}_{G}^{*}(K,-)$ and $\mathcal{I}_{G}^{*}(K,-)$ from $(\mathcal{K}(G), \mathrm{Ab}) \times O_{G}^{\mathrm{op}}$ to $\mathrm{Ab}^{*}$.

(ii) There exists a natural equivalence $z^{*}: \mathcal{I}_{G}^{*} \rightarrow H_{G}^{*}(-[-],-)$, and a natural transformation $\phi^{*}: \mathcal{I}_{G}^{*} \rightarrow \mathcal{H}_{G}^{*}$ of functors from $G$-CW ${ }^{\mathrm{op}} \times\left(O_{G}^{\mathrm{op}}, \mathrm{Ab}\right) \times$ $O_{G}^{\mathrm{op}}$, which is induced by the maps $\phi(G / H, K)$. 
(iii) For a generic coefficient system $M: O_{G}^{\mathrm{op}} \rightarrow \mathrm{Ab}$,

$$
\begin{gathered}
\mathcal{H}_{G}^{*}(K, M)(G / H) \cong H_{G}^{*}\left(G / H \times K, M \theta_{G}(G / H \times K)\right), \\
\mathcal{I}_{G}^{*}(K, M)(G / H) \cong H_{G}^{*}\left(G / H \times K, M \theta_{G}(K) p_{G / H}\right)
\end{gathered}
$$

and the map $\phi^{*}(K, M)(G / H)$ is induced by the natural transformation of local coefficient systems on $G / H \times K$

$$
\varphi\left(M, p_{G / H}\right): M \theta_{G}(K) p_{G / H} \rightarrow M \theta_{G}(G / H \times K) .
$$

Proof. (i) follows from 1.6 and 1.12. (ii) is a consequence of 1.7, 1.13 and the fact that the maps $\phi(G / H, K)$ form a natural transformation of functors from $O_{G} \times G$-CW to $G$-CW. (iii) follows from 1.13 and 1.8(ii) because

$$
\mathcal{I}_{G}^{*}(K, M)(G / H) \cong H_{G}^{*}\left(K[G / H], M \theta_{G}(K[G / H])\right)
$$

and $\phi(G / H, K)$ induces an isomorphism

$$
\begin{aligned}
H_{G}^{*}\left(K[G / H], M \theta_{G}(K[G / H])\right) & \\
& \rightarrow H_{G}^{*}\left(G / H \times K, M \theta_{G}(K[G / H]) \phi(G / H, K)\right),
\end{aligned}
$$

which satisfies the condition

$$
\begin{array}{r}
H_{G}^{*}(G / H \times K, \varphi(M, \phi(G / H, K))) H_{G}^{*}\left(\phi(G / H, K), M \theta_{G}(K[G / H])\right) \\
=H_{G}^{*}(\phi(G / H, K), M) .
\end{array}
$$

The equalities

$$
\begin{aligned}
M \theta_{G}(K) p_{G / H} & =M \theta_{G}(K[G / H]) \phi(G / H, K), \\
\varphi\left(M, p_{G / H}\right) & =\varphi(M, \phi(G / H, K))
\end{aligned}
$$

end the proof.

In Section 2 we shall prove the following result.

1.16. Corollary. The natural transformation of functors $\phi^{*}(-, M)(-)$ is an isomorphism if and only if $M$ is isomorphic to a constant functor. In this case, for any $G$-CW-complex $K$ and any subgroup $H$ of $G$,

$$
\begin{aligned}
\mathcal{I}_{G}^{*}(K, M)(G / H) & =H_{G}^{*}((G / H \times K) / G, M(G / G)) \\
& =H_{G}^{*}(K / H, M(G / G)) .
\end{aligned}
$$

Let $T$ be a Hecke functor. We shall prove that in this case the coefficient system $\mathcal{H}_{G}^{*}(K, T)$ can be extended to a Hecke functor, which will be denoted by the same symbol. This is a consequence of the following result.

1.17. Proposition. (i) Any functor $\kappa: \mathbb{H}_{G}^{\mathrm{op}} \times\left(\mathbb{H}_{G}^{\mathrm{op}}, \mathrm{Ab}\right) \rightarrow\left(O_{G}^{\mathrm{op}}, \mathrm{Ab}\right)$ induces a functor

$$
\kappa^{*}: G-\mathrm{CW} \times\left(\mathbb{H}_{G}^{\mathrm{op}}, \mathrm{Ab}\right) \times \mathbb{H}_{G}^{\mathrm{op}} \rightarrow \mathrm{Ab}^{*}
$$


such that, for every $G$-CW-complex $K$, every Hecke functor $T$, and every subgroup $H$ of $G$,

$$
\kappa^{*}(K, T)(G / H)=H_{G}^{*}(K, \kappa(G / H, T)) .
$$

(ii) If $\mathrm{d}: \kappa \rightarrow \kappa_{1}$ is a natural transformation of functors from $\mathbb{H}_{G}^{\mathrm{op}} \times$ $\left(\mathbb{H}_{G}^{\mathrm{op}}, \mathrm{Ab}\right)$ to $\left(O_{G}^{\mathrm{op}}, \mathrm{Ab}\right)$, then there exists a natural transformation of functors $\mathrm{d}^{*}: \kappa^{*} \rightarrow \kappa_{1}^{*}$ such that, for every $G$-CW-complex $K$, every Hecke functor $T$, and every subgroup $H$ of $G$,

$$
\mathrm{d}^{*}(K, T)(G / H)=H_{G}^{*}(K, \mathrm{~d}(G / H, T)) .
$$

The proof is easy and will be omitted. In Section 2 we shall prove the following result.

1.18. Proposition. There exists a functor $\Gamma: \mathbb{H}_{G}^{\mathrm{op}} \times\left(\mathbb{H}_{G}^{\mathrm{op}}, \mathrm{Ab}\right) \rightarrow$ $\left(O_{G}^{\mathrm{op}}, \mathrm{Ab}\right)$ and a natural equivalence $\psi^{*}: \Gamma^{*} \rightarrow \mathcal{H}_{G}^{*}$ of functors from $G-\mathrm{CW} \times$ $\left(\mathbb{H}_{G}^{\mathrm{op}}, \mathrm{Ab}\right) \times O_{G}^{\mathrm{op}}$ to $\mathrm{Ab}^{*}$. In particular, for every Hecke functor $T$, every $G$-CW-complex $K$ and every subgroup $H$ of $G$, there is an isomorphism

$$
\psi^{*}(K, T)(G / H): H_{G}^{*}(K, \Gamma(G / H, T)) \rightarrow \mathcal{H}_{G}^{*}(K, T)(G / H) .
$$

It is clear that using the isomorphism $\psi^{*}$ we can extend $\mathcal{H}_{G}^{*}(K, T)$ to a Hecke functor. If $f: \mathbb{Z}\left(G / H^{\prime}\right) \rightarrow \mathbb{Z}\left(G / H^{\prime \prime}\right)$ is a $\mathbb{Z}(G)$-module homomorphism, then

$$
\mathcal{H}_{G}^{*}(K, T)(f)=\psi^{*}(K, T)\left(G / H^{\prime}\right) H_{G}^{*}\left(K, \Gamma\left(f, \mathrm{id}_{T}\right)\right) \psi^{*}(K, T)\left(G / H^{\prime \prime}\right)^{-1} .
$$

Let $\gamma^{\prime}: \mathbb{H}_{G}^{\mathrm{op}} \times\left(\mathbb{H}_{G}^{\mathrm{op}}, \mathrm{Ab}\right) \rightarrow\left(O_{G}^{\mathrm{op}}, \mathrm{Ab}\right)$ be the composition of the functor $i^{\prime \prime}(\iota$, id $)$ which was defined before 1.1, and the restriction functor $i^{\prime}$ : $\left(\mathbb{H}_{G}^{\mathrm{op}}, \mathrm{Ab}\right) \rightarrow\left(O_{G}^{\mathrm{op}}, \mathrm{Ab}\right)$. Then $\gamma\left(\mathrm{id}, \mathrm{i}^{\prime}\right)=\gamma^{\prime}$ where $\gamma$ is the functor defined in 1.2, and for every Hecke functor $T$ and every subgroup $H$ of $G$,

$$
\mathcal{I}_{G}^{*}(K, T)(G / H)=H_{G}^{*}\left(K, \gamma^{\prime}(G / H, T)\right) .
$$

Thus $\mathcal{I}_{G}^{*}$ after restriction to the category $G$-CW $\times\left(\mathbb{H}_{G}^{\mathrm{op}}, \mathrm{Ab}\right) \times \mathbb{H}_{G}^{\mathrm{op}}$ is equal to $\left(\gamma^{\prime}\right)^{*}$.

1.19. Proposition. There exists a natural transformation $\zeta: \gamma^{\prime} \rightarrow \Gamma$ of functors from $\mathbb{H}_{G}^{\mathrm{op}} \times\left(\mathbb{H}_{G}^{\mathrm{op}}, \mathrm{Ab}\right)$ to $\mathrm{Ab}$ such that $\psi^{*} \zeta^{*}=\phi^{*}$ after restriction to $G-\mathrm{CW} \times\left(\mathbb{H}_{G}^{\mathrm{op}}, \mathrm{Ab}\right) \times O_{G}^{\mathrm{op}}$.

Proposition 1.19 will also be proved in Section 2. It implies that if $T$ is a Hecke functor, then the transformation $\phi^{*}(K, T): \mathcal{I}_{G}^{*}(K, T) \rightarrow \mathcal{H}_{G}^{*}(K, T)$ extends to a natural transformation of Hecke functors when $\mathcal{H}_{G}^{*}(K, T)$ is extended to a Hecke functor.

We shall need the following well known property of Hecke functors. Assume that $H$ is a subgroup of $G$ and that $H^{\prime}$ is a subgroup of $H$. Consider the $\mathbb{Z}(G)$-homomorphisms $a: \mathbb{Z}\left(G / H^{\prime}\right) \rightarrow \mathbb{Z}(G / H)$ and $a^{\prime}$ : 
$\mathbb{Z}(G / H) \rightarrow \mathbb{Z}\left(G / H^{\prime}\right)$ such that for every $g$ in $G, a\left(g H^{\prime}\right)=g H$ and $a^{\prime}(g H)=$ $\sum_{[h] \in H / H^{\prime}} g h H^{\prime}$.

1.20. Lemma. Let $T$ be a Hecke functor. Then there exist homomorphisms

$$
r\left(H, H^{\prime}\right): T(G / H) \rightarrow T\left(G / H^{\prime}\right), \quad i\left(H^{\prime}, H\right): T\left(G / H^{\prime}\right) \rightarrow T(G / H)
$$

such that the composition $i\left(H^{\prime}, H\right) r\left(H, H^{\prime}\right)$ is multiplication by $\left|H / H^{\prime}\right|$.

Proof. We define $r\left(H, H^{\prime}\right)=T(a)$ and $i\left(H^{\prime}, H\right)=T\left(a^{\prime}\right)$.

The following facts are immediate consequences of 1.20.

1.21. Corollary. (i) If $T\left(G / H^{\prime}\right)=(0)$, then $\left|H / H^{\prime}\right| T(G / H)=(0)$.

(ii) If $T\left(G / H^{\prime}\right)$ is a p-group and $\left|H / H^{\prime}\right|=p^{n}$, then $T(G / H)$ is a $p$ group.

(iii) Let $H_{p}$ be a Sylow p-subgroup of $H$ and let $T(G / H)_{p}$ be the $p$-torsion part of $T(G / H)$. Then $T(G / H)_{p}$ is a direct summand in $T\left(G / H_{p}\right)$.

(iv) If $T: \mathbb{H}_{G}^{\text {op }} \rightarrow \mathbb{Z}_{(p)}$-Mod is a p-local Hecke functor, then $T(G / H)$ is a direct summand in $T\left(G / H_{p}\right)$.

Assume now that $K$ is a $G$-CW-complex and that $L$ is a local coefficient system on $K$. Then there exists an equivariant cohomology theory $h^{*}: G-\mathrm{CW}^{\mathrm{op}} \rightarrow \mathrm{Ab}^{*}$ such that, for every $G$-CW-complex $K^{\prime}, h^{*}\left(K^{\prime}\right)=$ $H_{G}^{*}\left(P_{G}\left(K^{\prime}, K\right), L_{1}\right)$, where $L_{1}=L\left(p_{K^{\prime}}\right)_{1}$. The coefficient system of $h^{*}$ is equal to the restriction of $\mathcal{I}_{G}^{*}(K, L)$ to the category $O_{G}^{\text {op }}$. The results of [2], Ch. IV, imply the following fact.

1.22. Corollary. There exists an "Atiyah-Hirzebruch" spectral sequence

$$
H_{G}^{p}\left(K^{\prime}, \mathcal{I}_{G}^{q}(K, L)\right) \Rightarrow H_{G}^{p+q}\left(P_{G}\left(K^{\prime}, K\right), L_{1}\right)
$$

We shall study this spectral sequence in the case where the natural map $\left(p_{K^{\prime}}\right)_{1}: P_{G}\left(K^{\prime}, K\right) \rightarrow K$ induces an isomorphism of cohomology groups $H_{G}^{*}(K, L) \cong H_{G}^{*}\left(P_{G}\left(K^{\prime}, K\right), L_{1}\right)$.

1.23. Corollary. Assume that $M$ is a G-generic coefficient system such that, for every $k$ in $K$, the map $K^{\prime} / G_{k} \rightarrow \mathrm{pt}$, where $\mathrm{pt}$ is a one-point space, induces an isomorphism of cohomology groups

$$
M\left(G / G_{k}\right)=H^{*}\left(\mathrm{pt}, M\left(G / G_{k}\right)\right) \cong H^{*}\left(K^{\prime} / G_{k}, M\left(G / G_{k}\right)\right) .
$$

Then there exists a spectral sequence $H_{G}^{p}\left(K^{\prime}, \mathcal{I}_{G}^{q}(K, M)\right) \Rightarrow H_{G}^{p+q}(K, M)$.

Proof. There exists a spectral sequence

$$
E_{2}^{p, q}\left(K^{\prime}\right)=H_{G}^{p}\left(K, M^{q}\left(K^{\prime}\right)\right) \Rightarrow H_{G}^{p+q}\left(P_{G}\left(K^{\prime}, K\right), M\right),
$$


where the $M^{q}$ are $G$-generic coefficient systems such that, for every subgroup $H$ of $G$,

$$
\begin{aligned}
M^{q}\left(K^{\prime}\right)(G / H) & =H_{G}^{q}\left(P_{G}\left(K^{\prime}, G / H\right), M\right)=H_{G}^{q}\left(\left(K^{\prime} \times G / H\right) / G \times G / H, M\right) \\
& \cong H_{G}^{q}\left(K^{\prime} / H \times G / H, M\right) \cong H^{q}\left(K^{\prime} / H, M(G / H)\right) .
\end{aligned}
$$

It follows from the assumptions that $H_{G}^{*}\left(K, M^{q}\left(K^{\prime}\right)\right)=(0)$ for each natural number $q>0$, and that there is a natural transformation of functors $v\left(K^{\prime}\right)$ : $M^{*}\left(K^{\prime}\right) \rightarrow M^{0}\left(K^{\prime}\right)$ which induces an isomorphism $H_{G}^{*}\left(K, M^{*}\left(K^{\prime}\right)\right) \cong$ $H_{G}^{*}\left(K, M^{0}\left(K^{\prime}\right)\right)$.

Let $M^{*}=M^{*}(\mathrm{pt})$. Then $M^{q}=(0)$ whenever $q>0$, and the map $v(\mathrm{pt})$ : $M^{*} \rightarrow M^{0}$ is a natural equivalence of functors. The natural map $f_{K^{\prime}}: K^{\prime} \rightarrow$ pt induces a morphism of spectral sequences $E_{*}^{*, *}(\mathrm{pt}) \rightarrow E_{*}^{*, *}\left(K^{\prime}\right)$. Since $\left(p_{K^{\prime}}\right)_{1}=\left(p_{\mathrm{pt}}\right)_{1} P_{G}\left(f_{K^{\prime}}, \mathrm{id}_{K}\right)$ it follows that $\left(p_{K^{\prime}}\right)_{1}$ induces an isomorphism of cohomology groups $H_{G}^{p}(K, M) \cong H_{G}^{p}\left(P_{G}\left(K^{\prime}, K\right), M\right)$. We can now apply 1.22 .

We shall need the following notation. Assume that $F$ is a $G$-set of subgroups of $G$, i.e. a set of subgroups closed under conjugation by elements of $G$. Then we shall denote by $F_{p}$ the set of all Sylow $p$-subgroups of groups in $F$. If $F, F_{0}$ are $G$-sets of subgroups of $G$ then the $G$-set $\left\{H \cap H_{0}\right.$ : $\left.H \in F, H_{0} \in F_{0}\right\}$ will be denoted by $F \square F_{0}$. We shall denote by $F \cup F_{0}$ the ordinary union of the sets $F$ and $F_{0}$. We shall also use the notation $F^{\prime}=F \cup\{G\}$. If $K$ is a $G$-CW-complex, then $F(K)=\left\{G_{k}: k \in K\right\}$.

Assume now that $R$ is a commutative ring. We say that a $\mathrm{CW}$-complex $K_{0}$ is $R$-acyclic if the map $\Pi: K_{0} \rightarrow G / G$ induces an $R$-module isomorphism $H_{*}\left(K_{0}, R\right) \rightarrow R$ of the ordinary cellular homology groups with $R$ as coefficients. This is equivalent to the condition that, as $R$-modules, $H_{*}\left(K_{0}, R\right)$ is isomorphic to $H^{*}\left(K_{0}, R\right)$ and to $R$.

1.24. Lemma. (i) Let $M: O_{G}^{\mathrm{op}} \rightarrow R$-Mod be a generic $G$-coefficient system. Assume that for every subgroup $H \in F(K)^{\prime}$ the $\mathrm{CW}$-complex $K^{H}$ is $R$-acyclic. Then $\Pi$ induces an isomorphism $M(G / G) \cong H_{G}^{*}(K, M)$.

(ii) Assume that, for every subgroup $H \in F(K)^{\prime}$, $\Pi$ induces an isomorphism $\mathrm{h}_{*}(K, R)(G / H) \rightarrow R$, where $\mathrm{h}_{n}(K, R)(G / H)=H_{n}\left(C_{*}(K, R)^{H}\right)$. Then, for every Hecke functor $T: \mathbb{H}_{G}^{\mathrm{op}} \rightarrow R$-Mod, $\Pi$ induces an isomorphism $T(G / G) \cong H_{G}^{*}(K, T)$.

This result will be proved in Section 2.

1.25. LemmA. Let $F$ be a $G$-set of subgroups of $G$ and let $K$ be a $G$-CWcomplex. Assume that one of the following conditions holds.

(i) The CW-complex $K^{H}$ is $R$-acyclic whenever $H$ belongs to $F(K)^{\prime} \square F$ $=(F(K) \square F) \cup F$. 
(ii) There exists a prime number $p$ such that $\left|G / G_{p}\right|$ is an invertible element of $R$ and the $\mathrm{CW}$-complex $K^{H}$ is $R$-acyclic whenever $H$ belongs to $F(K)^{\prime} \square F_{p}$.

Then for every $H$ in $F$, the $\mathrm{CW}$-complex $K / H$ is $R$-acyclic and $\Pi$ induces an isomorphism $\mathrm{h}_{*}(K, R)(G / H) \rightarrow R$.

Proof. It is sufficient to prove that $\Pi$ induces an isomorphism

$$
\mathrm{h}_{*}(K, R)(G / H) \rightarrow R,
$$

because, for each natural number $n$, there is an isomorphism

$$
\text { b : } H_{n}\left(C_{*}(K / H, R)\right) \rightarrow H_{n}\left(C_{*}(K, R)^{H}\right) \text {. }
$$

If (i) holds, then from 1.24(i) it follows that $\Pi$ induces an isomorphism $R \rightarrow H^{0}\left(\operatorname{Hom}_{R}\left(C_{*}(K, R)^{H}, R\right)\right)$ and that, for each $n>0$,

$$
H^{n}\left(\operatorname{Hom}_{R}\left(C_{*}(K, R)^{H}, R\right)\right)=(0) .
$$

This implies that $h_{*}(\Pi, R)$ is an isomorphism.

Assume now that (ii) holds. Let $H \in F$ and let $H_{p}$ be a Sylow $p$ subgroup of $H$. Condition (i) of this lemma holds for $F=F_{p}$, hence $\Pi$ induces an isomorphism $h_{*}(K, R)\left(G / H_{p}\right) \rightarrow R$. For each $n, h_{n}(K, R)$ is a Hecke functor and $h_{n}(\Pi, R)$ is a natural transformation of Hecke functors. The result now follows from 1.20 and from the assumption that $\left|H / H_{p}\right|$ is an invertible element of $R$.

1.26. Corollary. Let $K$ and $K^{\prime}$ be $G$-CW-complexes. Assume that one of the conditions of 1.25 holds for $K^{\prime}$ and the family $F=F(K)$. Then, for any coefficient system $M: O_{G}^{\mathrm{op}} \rightarrow R$-Mod, there exists a spectral sequence

$$
H_{G}^{p}\left(K^{\prime}, \mathcal{I}_{G}^{q}(K, M)\right) \Rightarrow H_{G}^{p+q}(K, M) .
$$

Proof. Lemma 1.25 implies that $K^{\prime} / H$ is $R$-acyclic for each $H$ in $F$. Thus, by the Universal Coefficient Theorem for cohomology,

$$
H^{*}(K / H, M(G / H))=H^{0}(K / H, M(G / H))=M(G / H)
$$

for each $H$ in $F$. Now we can apply 1.23 .

As an immediate consequence of 1.26 we obtain the following fact.

1.27. Corollary. Let $p$ be a prime and let $K$ be a $G$-CW-complex such that, for each $k$ in $K$, the Sylow p-subgroups of $G_{k}$ are equal to $(e)$. Assume that $\left|G / G_{p}\right|^{-1} \in R$. Then there exists a spectral sequence

$$
H^{p}\left(G, H_{G}^{q}(K, M[G /(e)])\right) \Rightarrow H_{G}^{p+q}(K, M) .
$$

Pr o of. In this case we may consider $K^{\prime}$ equal to a universal free $G$-CWcomplex $E G$. The Bredon cohomology groups of this complex with respect to any coefficient system $M^{\prime}$ are equal to the cohomology groups of $G$ with 
coefficients in the $\mathbb{Z}(G)$-module $M^{\prime}(G /(e)$ ). (See Example 1 on p. I-25 of [2].)

The following result shows that in many cases the spectral sequence described in 1.26 trivializes.

1.28. Corollary. Let $R$ be a commutative ring and let $K^{\prime}$ be a $G$-CWcomplex. Assume that one of the following conditions holds.

(i) There exists a prime number $p$ such that $K^{\prime H}$ is $R$-acyclic whenever $H \in\left(F\left(K^{\prime}\right)^{\prime}\right)_{p} \square F\left(K^{\prime}\right)^{\prime}$ and $\left|G / G_{p}\right|$ is an invertible element of $R$.

(ii) For every prime number $p, K^{\prime H}$ is $\mathbb{Z} / p$-acyclic whenever $H \in$ $\left(F\left(K^{\prime}\right)^{\prime}\right)_{p}$ 口 $F\left(K^{\prime}\right)^{\prime}, K$ is $\mathbb{Z}$-acyclic and $R=\mathbb{Z}$.

(iii) The CW-complex $K^{\prime}$ is finite-dimensional, $\mathbb{Z}$-acyclic and $R=\mathbb{Z}$.

(iv) The $\mathrm{CW}$-complex $K^{\prime}$ is finite-dimensional, $\mathbb{Z} / p$-acyclic and $R=\mathbb{Z} / p$.

Then, for any Hecke functor $T: \mathbb{H}_{G}^{\mathrm{op}} \rightarrow R$-Mod,

$$
T(G / G)=H_{G}^{0}\left(K^{\prime}, T\right)=H_{G}^{*}\left(K^{\prime}, T\right) .
$$

Pro of. If (i) holds, then the result follows from 1.24(ii) and from 1.25. Assume that (ii) holds. Lemma 1.25 implies that, for each prime $p$ and each $H \in F\left(K^{\prime}\right)^{\prime}, \mathrm{h}_{*}(\Pi, \mathbb{Z} / p)(G / H)$ is an isomorphism. Assume that $H \in$ $F\left(K^{\prime}\right)^{\prime}$. From the fact that $h_{n}\left(K^{\prime}, \mathbb{Z}\right)$ is a Hecke functor and from 1.21(i) it follows that

$$
|H| \text { Coker }_{0}(\Pi, \mathbb{Z})(G / H)=|H| \operatorname{Ker}_{0}(\pi, \mathbb{Z})(G / H)=(0),
$$

and $|H| \mathrm{h}_{n}\left(K^{\prime}, \mathbb{Z}\right)(G / H)=(0)$ for each $n>0$, because $H_{n}\left(K^{\prime}, \mathbb{Z}\right)=(0)$. Hence $h_{*}(\Pi, \mathbb{Z})(G / H)$ is an isomorphism and we can apply 1.24(ii). The statements (iii) and (iv) are consequences of the Smith theory ([3], Ch. III. $5.2)$. In this case $\mathbb{Z} / p$-acyclicity of $K^{\prime}$ implies $\mathbb{Z} / p$-acyclicity of $K^{\prime H}$, for every $p$-subgroup $H$ of $G$.

For a $G$-set $F$ of subgroups of $G$, we shall denote by $O(F)$ the full subcategory of $O_{G}$ whose objects are the $G$-orbits $G / H$ where $H$ belongs to $F$. The following result can be considered as a special case of one of the results of [8], which says that every $p$-local Mackey functor is acyclic on the category $O(F)$ in the case where $F$ contains the Sylow $p$-subgroups of $G$ and $F \square F \subseteq F$.

1.29. Corollary. Let $F$ be a $G$-set of subgroups of $G$ such that $F \square F$ $\subseteq F, F^{\prime} \square F_{p} \subseteq F$ and $F_{p}=\left(F^{\prime}\right)_{p}$, i.e. all Sylow p-subgroups of $G$ belong to $F_{p}$. Assume that $\left|G / G_{p}\right|^{-1} \in R$. Then, for any Hecke functor $T: \mathbb{H}_{G}^{\mathrm{op}} \rightarrow$ $R$-Mod,

$$
T(G / G)=\lim _{O(F)}^{*} T=\lim _{O(F)}^{0} T .
$$


Proof. Let $F_{m}$ be the set of maximal subgroups of $F$. Assume that $F_{m}=\left\{H_{0}, \ldots, H_{n}\right\}$. Define $E F=*_{i=0}^{s} E\left(G / H_{i}\right)$ where $E(G / H)=$ $*_{i=0}^{\infty} G / H$. It is well known that $E F$ has a structure of a $G$-CW-complex such that, for every $H \in F, E F^{H}$ is $R$-acyclic and $F(E F) \subseteq F$, and that $([10],[13]) H_{G}^{*}(E F, T)=\lim _{O(F)}^{*} T$. Now, it is sufficient to apply 1.28 .

2. Hecke structure on categories associated to $G$-posets. In this section, we shall prove the results which were stated and not proved in Section 1. Some of these will be considered as special cases of more general facts concerning categories associated to $G$-posets which will be presented in this section.

Let $W$ be a $G$-poset, i.e. $W$ is a poset and $G$ acts on $W$ in such a way that, for any $g$ in $G$ and any $w, w^{\prime}$ in $W, w \subseteq w^{\prime}$ implies that $g w \subseteq g w^{\prime}$.

The category $W[G]$ is defined as follows. Its objects are the elements of $W$; for any $w, w^{\prime}$ in $W$

$$
\operatorname{Mor}_{W[G]}\left(w^{\prime}, w\right)=\left\{g \in G: g w^{\prime} \subseteq w\right\},
$$

and the composition of morphisms is the multiplication in $G$. Let $G_{w}=$ $\{g \in G: g w=w\}$. Then $G_{w}=\operatorname{Mor}_{W[G]}(w, w)$ and $\operatorname{Mor}_{W[G]}\left(w^{\prime}, w\right)$ is a left $G_{w^{-}}$set and a right $G_{w^{\prime}}$-set.

By Sub- $G$ we shall denote the $G$-poset of all subgroups of $G$. The group $G$ acts on $W[G]$ by conjugation. Assume that $d: W \rightarrow \operatorname{Sub}-G$ is a $G$-poset map such that, for every $w$ in $W, d w$ is a subgroup of $G_{w}$. Such $G$-poset maps will be called admissible. By $W_{d}[G]$ we shall denote the category whose objects are the elements of $W$ and whose morphism sets are obtained from the morphism sets of $W[G]$ in such a way that, for any $w, w^{\prime}$ in $W$,

$$
\operatorname{Mor}_{W_{d}[G]}\left(w^{\prime}, w\right)=\operatorname{Mor}_{W[G]}\left(w^{\prime}, w\right) / d w .
$$

The composition of morphisms in $W_{d}[G]$ is induced by the composition of morphisms in $W[G]$. This definition is correct because if $g w^{\prime} \subseteq w$, then $g\left(d w^{\prime}\right) g^{-1} \subseteq d w$ so we have the inclusion $g d w^{\prime} \subseteq(d w) g$ of subsets of $G$. The morphism $w^{\prime} \rightarrow w$ of $W_{d}[G]$ which is defined by an element $g$ of $G$ such that $g w^{\prime} \subseteq w$ will be denoted by $[g]$.

If $H$ is a subgroup of $G$ then any $G$-poset can be considered as an $H$ poset. Assume that $d: W \rightarrow$ Sub- $G$ is an admissible $G$-poset map. Then $d_{H}: W \rightarrow$ Sub- $H$ will denote the admissible $H$-poset map such that, for every $w$ in $W, d_{H} w=H \cap d w$. The category $W_{d_{H}}[H]$ will be denoted by $W_{d}[H]$ and will be considered as a subcategory of $W_{d}[G]$.

If $H=(e)$, where $e$ is the neutral element of $G$, then we obtain the category associated to the poset $W$. We shall use the notation $W_{d}[(e)]=W$. If $V$ is a $G$-subposet of $W$, then the restriction of $d$ to $V$ will also be denoted by $d$. 
Now we present the main examples of categories described above.

2.1. ExAmple. Let $W=$ Sub- $G$ and let $d=\mathrm{id}:$ Sub- $G \rightarrow$ Sub- $G$. Then $W_{d}[G]=O_{G}$. The category $W_{d}[H]$ will be denoted by $O_{G, H}$. Its objects are the $G$-orbits $G / H^{\prime}$ and its morphisms are those $G$-maps $f: G / H^{\prime} \rightarrow G / H^{\prime \prime}$ which are defined by the elements $h$ of $H$ satisfying the condition $h^{-1} H^{\prime} h \subseteq$ $H^{\prime \prime}$ in such a way that $f\left(g H^{\prime}\right)=g h H^{\prime \prime}$.

2.2. Example. Assume that $K$ is a $G$-CW-complex. Let $V^{\prime}$ be the $G$-poset of all finite subcomplexes of $K$ and let $W^{\prime}=V^{\prime \text { op }}$ be the $G$-poset opposite to $V^{\prime}$. For every finite subcomplex $K^{\prime}$ of $K$, we define

$$
d\left(K^{\prime}\right)=G_{K^{\prime}}=\left\{g \in G: g k=k \text { whenever } k \in K^{\prime}\right\} .
$$

Then $W_{d}^{\prime}[H]^{\text {op }}=\mathcal{K}_{H}$ for any subgroup $H$ of $G$. If $V$ is the $G$-poset of all subcomplexes of $K$ which have the form $K(s)$, where $s$ is a cell of $K$, then $W_{d}[H]^{\mathrm{op}}=\mathcal{K}(H)$, where $W=V^{\mathrm{op}}$.

Let $\theta_{d}(G): W_{d}[G] \rightarrow O_{G}$ be the covariant functor such that, for any $w$ in $W, \theta_{d}(G)(w)=G / d w$ and, for any morphism $[g]: w^{\prime} \rightarrow w$ of $W_{d}[G]$, $\theta_{d}(G)([g])=[g]$. Assume that

$$
\kappa_{d}(G): \mathbb{H}_{G}^{\mathrm{op}} \times\left(W_{d}[G]^{\mathrm{op}}, \mathrm{Ab}\right) \rightarrow\left(W_{d}[G]^{\mathrm{op}}, \mathrm{Ab}\right)
$$

is the functor $\left(\iota \mathrm{i} \theta_{d}(G)\right)^{\prime \prime}(\iota, \mathrm{id})$ which was defined before Proposition 1.1. For any subgroup $H$ of $G$ and any contravariant functor from $W_{d}[G]$ to $\mathrm{Ab}$,

$$
\kappa_{d}(G)(G / H, M)(w)=\operatorname{Hom}_{\mathbb{Z}(G)}(\mathbb{Z}(G / H \times G / d w), \mathbb{Z}) \otimes M(w) .
$$

In Example 2.1, $\kappa_{d}(G)$ is the functor $\gamma$ defined in 1.2. In Example 2.2, we obtain the functor $\gamma_{K}$ described in 1.4. We shall also use the notation $\kappa_{d}(G)(G / H, M)=M[G / H]$.

Assume now that $M$ and $N$ are contravariant functors from $W_{d}[G]$ to Ab. For any subgroup $H$ of $G$, let $\lambda(H, G): W_{d}[H] \rightarrow W_{d}[G]$ be the inclusion functor.

The $\mathbb{Z}$-module of all natural transformations of $N \lambda(H, G)$ to $M \lambda(H, G)$ will be denoted by $\operatorname{Hom}_{W_{d}[H]}(N, M)$. The group $G$ acts on the $\mathbb{Z}$-module $\operatorname{Hom}_{W}(N, M)$, where $W=W_{d}[(e)]$, in such a way that for any $\varrho: N \rightarrow M$ in $\operatorname{Hom}_{W}(N, M)$, and for $w \in W$ and $g \in G$,

$$
(g \varrho)(w)=M\left([g]^{-1}\right) \varrho\left(g^{-1} w\right) N([g]),
$$

where $[g]: g^{-1} w \rightarrow w$.

2.3. Proposition. The image of the group monomorphism

$$
\mu: \operatorname{Hom}_{W_{d}[H]}(N, M) \rightarrow \operatorname{Hom}_{W}(N, M)
$$

is equal to $\operatorname{Hom}_{W}(N, M)^{H}$.

Proof. It is obvious that the image of $\mu$ is contained in $\operatorname{Hom}_{W}(N, M)^{H}$. Let $\tau$ be a natural transformation from $N \lambda(e, H)$ to $M \lambda(e, H)$. Assume 
that, for every $w$ in $W$ and $h$ in $H$,

$$
M([h]) \tau(w)=\tau\left(h^{-1} w\right) N([h]),
$$

where $[h]: h^{-1} w \rightarrow w$ is an isomorphism of $W_{d}[H]$. Let $\left[h^{\prime}\right]: w^{\prime} \rightarrow w$ be an arbitrary morphism of $W_{d}[H]$. Then $\left[h^{\prime}\right]$ is the composition of the morphism $[e]: w^{\prime} \rightarrow h^{\prime-1} w$ of $W=W_{d}[e]$ and the isomorphism $\left[h^{\prime}\right]: h^{\prime-1} w \rightarrow w$ of $W_{d}[H]$. Hence $M\left(\left[h^{\prime}\right]\right) \tau(w)=\tau\left(w^{\prime}\right) N\left(\left[h^{\prime}\right]\right)$, which ends the proof.

We shall need the following facts, which can be obtained immediately from the results of Auslander [1] and Mitchell [9]. Let $\mathbb{C}$ be a small category. Consider two functors $P: \mathbb{C} \rightarrow \mathrm{Ab}$ and $Q: \mathbb{C}^{\mathrm{op}} \rightarrow \mathrm{Ab}$. Their tensor product over $\mathbb{C}, P \otimes_{\mathbb{C}} Q$, is defined as the coequalizer of the diagram

$$
\coprod_{B, B^{\prime} \in \mathrm{Ob} \mathbb{C}} \coprod_{f \in \operatorname{Mor}_{\mathbb{C}}\left(B, B^{\prime}\right)} P(B) \otimes Q\left(B^{\prime}\right) \underset{t^{\prime}}{\stackrel{t}{\rightrightarrows}} \coprod_{B^{\prime \prime} \in \mathrm{Ob} \mathbb{C}} P\left(B^{\prime \prime}\right) \otimes Q\left(B^{\prime \prime}\right),
$$

where $t(p \otimes q)=p \otimes Q(f) q$ and $t^{\prime}(p \otimes q)=P(f) p \otimes q$ whenever $p \in P(B)$ and $q \in Q\left(B^{\prime}\right)$. Thus $P \otimes_{\mathbb{C}} Q$ is the coend of the functor $\mathcal{P}: \mathbb{C} \times \mathbb{C}^{\text {op }} \rightarrow$ Ab such that $\mathcal{P}\left(B, B^{\prime}\right)=P(B) \otimes Q\left(B^{\prime}\right)$. If $M$ and $N$ are contravariant functors from $\mathbb{C}$ to $\mathrm{Ab}$ then we denote by $\operatorname{Hom}_{\mathbb{C}}(M, N)$ the abelian group of all additive natural transformations from $M$ to $N$. This group can also be considered as the end of an appropriate functor.

Let $B$ be an object of $\mathbb{C}$. Then the representable functor $\mathbb{Z}\left(\operatorname{Mor}_{\mathbb{C}}(-, B)\right)$ is a projective object in the category $\left(\mathbb{C}^{\mathrm{op}}, \mathrm{Ab}\right)$ of contravariant functors from $\mathbb{C}$ to $\mathrm{Ab}$. For any functor $N: \mathbb{C}^{\mathrm{op}} \rightarrow \mathrm{Ab}$ there is a group isomorphism

$$
\operatorname{Hom}_{\mathbb{C}}\left(\mathbb{Z}\left(\operatorname{Mor}_{\mathbb{C}}(-, B)\right), N\right)=N(B),
$$

which is natural in $B$.

Assume that $\mathbb{C}^{\prime}$ is a subcategory of $\mathbb{C}$ and that $M$ is a contravariant functor from $\mathbb{C}^{\prime}$ to $\mathrm{Ab}$. Then $\mathbb{C} \otimes \mathbb{C}^{\prime} M$ and $\operatorname{Hom}_{\mathbb{C}^{\prime}}(\mathbb{C}, M)$ are contravariant functors from $\mathbb{C}$ to $\mathrm{Ab}$ such that, for any functor $N: \mathbb{C}^{\mathrm{op}} \rightarrow \mathrm{Ab}$,

$$
\begin{aligned}
\operatorname{Hom}_{\mathbb{C}}\left(\mathbb{C} \otimes_{\mathbb{C}^{\prime}} M, N\right) & =\operatorname{Hom}_{\mathbb{C}^{\prime}}(M, N), \\
\operatorname{Hom}_{\mathbb{C}}\left(N, \operatorname{Hom}_{\mathbb{C}^{\prime}}(\mathbb{C}, M)\right) & =\operatorname{Hom}_{\mathbb{C}^{\prime}}(N, M) .
\end{aligned}
$$

For any object $B$ of $\mathbb{C}$ we have

$$
\begin{aligned}
\left(\mathbb{C} \otimes_{\mathbb{C}^{\prime}} M\right)(B) & =\mathbb{Z}\left(\operatorname{Mor}_{\mathbb{C}}(B,-)\right) \otimes_{\mathbb{C}^{\prime}} M, \\
\left(\operatorname{Hom}_{\mathbb{C}^{\prime}}(\mathbb{C}, M)\right)(B) & =\operatorname{Hom}_{\mathbb{C}^{\prime}}\left(\mathbb{Z}\left(\operatorname{Mor}_{\mathbb{C}}(-, B), M\right) .\right.
\end{aligned}
$$

It is obvious that $\mathbb{C} \otimes_{\mathbb{C}^{\prime}} M$ (resp. $\operatorname{Hom}_{\mathbb{C}^{\prime}}(\mathbb{C}, M)$ ) is a left (resp. right) Kan extension of the functor $M$ along the inclusion $\mathbb{C}^{\prime} \rightarrow \mathbb{C}$. It follows from the definition that $\left(\mathbb{C} \otimes_{\mathbb{C}^{\prime}} M\right)(B)$ is the coend of the functor $\mathcal{P}: \mathbb{C}^{\prime} \times \mathbb{C}^{\prime o p} \rightarrow \mathrm{Ab}$ such that

$$
\mathcal{P}\left(B^{\prime}, B^{\prime \prime}\right)=\mathbb{Z}\left(\operatorname{Mor}_{\mathbb{C}}\left(B, B^{\prime}\right)\right) \otimes M\left(B^{\prime \prime}\right)
$$


and that $\operatorname{Hom}_{\mathbb{C}^{\prime}}(\mathbb{C}, M)(B)$ is the end of the functor $\mathcal{P}^{\prime}: \mathbb{C}^{\prime} \times \mathbb{C}^{\prime \text { op }} \rightarrow \mathrm{Ab}$ such that

$$
\mathcal{P}^{\prime}\left(B^{\prime}, B^{\prime \prime}\right)=\operatorname{Hom}\left(\mathbb{Z}\left(\operatorname{Mor}_{\mathbb{C}}\left(B^{\prime}, B\right)\right), M\left(B^{\prime \prime}\right)\right) .
$$

Assume now that $\mathbb{C}=W_{d}[G]$ and $\mathbb{C}^{\prime}=W$. For any functor $M$ : $W_{d}[G]^{\mathrm{op}} \rightarrow \mathrm{Ab}$, we can consider $\operatorname{Hom}_{W}\left(W_{d}[G], M\right)$ as a contravariant functor from $W_{d}[G]$ to $\mathbb{Z}(G)$-Mod. The action of $G$ on $\operatorname{Hom}_{W}\left(W_{d}[G], M\right)(w)$ is given by the action of $G$ on the group of natural transformations

$$
\operatorname{Hom}_{W}\left(\mathbb{Z}\left(\operatorname{Mor}_{W_{d}[G]}(-, w)\right), M\right) \text {. }
$$

Let $\vartheta: \mathbb{Z}(G)$-Mod $\rightarrow\left(\mathbb{H}_{G}^{\text {op }}, \mathrm{Ab}\right)$ be the composite of $\iota: \mathbb{H}_{G} \rightarrow \mathbb{Z}(G)$-Mod and the Yoneda functor $\mathbb{Z}(G)$-Mod $\rightarrow\left(\mathbb{Z}(G)\right.$-Mod $\left.{ }^{\mathrm{op}}, \mathrm{Ab}\right)$. We define a functor

$$
\nu_{d}(G): \mathbb{H}_{G}^{\mathrm{op}} \times\left(W_{d}[G]^{\mathrm{op}}, \mathrm{Ab}\right) \rightarrow\left(W_{d}[G]^{\mathrm{op}}, \mathrm{Ab}\right)
$$

in such a way that

$$
\nu_{d}(G)(G / H, M)(w)=\vartheta\left(\operatorname{Hom}_{W}\left(W_{d}[G], M\right)(w)\right)(G / H) .
$$

2.4. Corollary. Let $H$ be a subgroup of $G$ and let $M$ be a contravariant functor from $W_{d}[G]^{\mathrm{op}}$ to $\mathrm{Ab}$. Then

$$
\nu_{d}(G)(G / H, M)=\operatorname{Hom}_{W}\left(W_{d}[G], M\right)^{H}=\operatorname{Hom}_{W_{d}[H]}\left(W_{d}[G], M\right) .
$$

Proof. Let $w \in W$. Then Proposition 2.3 implies that

$$
\begin{aligned}
\operatorname{Hom}_{W}\left(W_{d}[G], M\right)(w)^{H} & =\operatorname{Hom}_{W}\left(\mathbb{Z}\left(\operatorname{Mor}_{W_{d}[G]}(-, w)\right), M\right)^{H} \\
& =\operatorname{Hom}_{W_{d}[H]}\left(\mathbb{Z}\left(\operatorname{Mor}_{W_{d}[G]}(-, w)\right), M\right) .
\end{aligned}
$$

2.5. Proposition. (i) There exists a natural equivalence of functors $\mathrm{t}$ : $\kappa_{d}(G) \rightarrow \nu_{d}(G)$.

(ii) For any functors $N, M: W_{d}[G]^{\mathrm{op}} \rightarrow$ Ab there exists an isomorphism

$$
\operatorname{Hom}_{W_{d}[G]}(N, M[G / H]) \rightarrow \operatorname{Hom}_{W}(N, M)^{H},
$$

which is natural in $N$ and $M$. This equivalence is also natural with respect to $G / H$ in $\mathbb{H}_{G}$.

Proof. (i) It follows immediately from the definition that, for any $w$ in $W$,

$$
\mathbb{Z}\left(\operatorname{Mor}_{W_{d}[G]}(-, w)\right)=\coprod_{[g] \in G / d w} \mathbb{Z}\left(\operatorname{Mor}_{W}(-, g w)\right) .
$$

Assume that $[g] \in G / d w$ and that $w^{\prime} \subseteq g w$. Then the morphism $w^{\prime} \subseteq g w$ of $W$ corresponds to the morphism $\left[g^{-1}\right]: w^{\prime} \rightarrow w$ of $W_{d}[G]$. Hence

$$
\nu_{d}(G)(G / H, M)(w)=\left(\prod_{[g] \in G / d w} M(g w)\right)^{H} .
$$


The group $G$ acts on $\prod_{[g] \in G / d w} M(g w)$ in such a way that, for any $g^{\prime} \in G$, we have

$$
g^{\prime}\left(\prod_{[g] \in G / d w} m(g w)\right)=\prod_{[g] \in G / d w} M\left(\left[\left(g^{\prime}\right)^{-1}\right]\right) m\left(\left(g^{\prime}\right)^{-1} g w\right),
$$

where, for every $g \in G,\left[g^{\prime-1}\right]: g w \rightarrow\left(g^{\prime}\right)^{-1} g w$.

Let $\xi \otimes m$ be an element of $\kappa_{d}(G)(G / H, M)(w)$ such that $\xi: \mathbb{Z}(G / H \times$ $G / d w) \rightarrow \mathbb{Z}$ is a $\mathbb{Z}(G)$-module homomorphism and $m$ belongs to $M(w)$. We define

$$
\mathrm{t}(G / H, M)(w)(\xi \otimes m)=\prod_{[g] \in G / d w} \xi(H, g d w) M\left(\left[g^{-1}\right]\right) m,
$$

where $\left[g^{-1}\right]: g w \rightarrow w$. It is easy to check that this definition is correct and that $\mathrm{t}$ is a natural equivalence of functors. For example, let $\mathrm{n}: \mathbb{Z}\left(G / H_{1}\right) \rightarrow \mathbb{Z}(G / H)$ be the $\mathbb{Z}(G)$-module homomorphism such that $\mathrm{n}\left(H_{1}\right)=\sum_{\left[g^{\prime}\right] \in G / H} n\left(\left[g^{\prime}\right]\right) g^{\prime} H$. Then

$$
\xi(\mathrm{n} \otimes \mathrm{id})\left(H_{1}, g d w\right)=\sum_{\left[g^{\prime}\right] \in G / H} n\left(\left[g^{\prime}\right]\right) \xi\left(g^{\prime} H, g d w\right)
$$

and $\nu_{d}(G)\left(\mathrm{n}, \mathrm{id}_{M}\right)(w)$ is equal to the operation by $\sum_{\left[g^{\prime}\right] \in G / H} n\left(\left[g^{\prime}\right]\right)\left(g^{\prime}\right)$. Thus

$$
\begin{aligned}
& \left(\mathrm{t}\left(G / H_{1}, M\right) \kappa_{d}\left(\mathrm{n}, \operatorname{id}_{M}\right)\right)(w)(\xi \otimes m)=\mathrm{t}\left(G / H_{1}, M\right)(w)(\xi(\mathrm{n} \otimes \mathrm{id}) \otimes m) \\
& \quad=\prod_{[g] \in G / d w} \sum_{\left[g^{\prime}\right] \in G / H} n\left(\left[g^{\prime}\right]\right) \xi\left(g^{\prime} H, g d w\right) M\left(\left[g^{-1}\right]\right) m \\
& \quad=\prod_{[g] \in G / d w} \sum_{\left[g^{\prime}\right] \in G / H} n\left(\left[g^{\prime}\right]\right) \xi\left(H,\left(g^{\prime}\right)^{-1} g d w\right) M\left(\left[g^{-1}\right]\right) m \\
& \quad=\sum_{\left[g^{\prime}\right] \in G / H} n\left(\left[g^{\prime}\right]\right) \prod_{[g] \in G / d w} M\left(\left[\left(g^{\prime}\right)^{-1}\right]\right) \xi\left(H,\left(g^{\prime}\right)^{-1} g H\right) M\left(\left[g^{-1} g^{\prime}\right]\right) m \\
& \quad=\nu_{d}(G)\left(\mathrm{n}, \operatorname{id}_{M}\right)(w) \prod_{[g] \in G / d w} \xi(H, g d w) M\left(\left[g^{-1}\right]\right) m \\
& \left.\quad=\nu_{d}(G)\left(\mathrm{n}, \operatorname{id}_{M}\right) \mathrm{t}(G / H, M)\right)(w)(\xi \otimes m) .
\end{aligned}
$$

This proves that $\mathrm{t}(G / H, M)$ is a natural transformation with respect to $G / H$ in $\mathbb{H}_{G}$.

(ii) follows from (i), 2.3 and 2.4.

Assume now that $C=C_{*}$ is a chain complex of contravariant functors from $W_{d}[G]$ to Ab. For any functor $M: W_{d}[G]^{\mathrm{op}} \rightarrow \mathrm{Ab}$, we denote by $H^{n}(C, M)$ the $n$th cohomology group of the cochain complex 
$\operatorname{Hom}_{W_{d}[G]}\left(C_{*}, M\right)$. As an immediate consequence of $2.5($ ii), we obtain the following result, which will be used in the proof of Proposition 1.6.

2.6. Corollary. There exists an isomorphism of contravariant functors from $\mathbb{H}_{G}$ to $\mathrm{Ab}^{*}$

$$
H^{*}(C, M[-]) \rightarrow \mathrm{h}\left(\operatorname{Hom}_{W}\left(C_{*}, M\right),-\right),
$$

which is natural in $M$ and $C$.

We shall now give another description of the cochain complex of Bredon cohomology. In the case of cohomology with a generic coefficient system this was done by Bredon in [2], Ch. I.9. Let $K$ be a $G$-CW-complex. Then $c_{*}(K)$ denotes the chain complex of functors from $O_{G}^{\text {op }}$ to Ab such that, for any subgroup $H$ of $G, c_{*}(K)(G / H)$ is equal to the ordinary cellular chain complex of $K^{H}$ with $\mathbb{Z}$ as coefficients. If $n$ is a natural number, then

$$
c_{n}(K)=\mathbb{Z}\left(\operatorname{Map}_{G}\left(-, S_{n} K\right)\right)=\coprod_{[s] \in\left(S_{n} K\right) / G} \mathbb{Z}\left(\operatorname{Mor}_{O_{G}}\left(-, G / G_{s}\right)\right),
$$

where $S_{n} K$ is the $G$-set of $n$-cells of $K$ and $\operatorname{Map}_{G}(-,-)$ denotes the set of $G$-maps. For any generic coefficient system $M$ there is an isomorphism of cochain complexes

$$
C^{*}(K, M)^{G}=\operatorname{Hom}_{O_{G}}\left(c_{*}(K), M\right) .
$$

Let $\lambda^{\prime}$ denote the inclusion $O_{G,(e)} \rightarrow O_{G}$. One can easily check that

$$
c_{n}(K) \lambda^{\prime}=\coprod_{s \in S_{n} K} \mathbb{Z}\left(\operatorname{Mor}_{O_{G,(e)}}\left(-, G / G_{s}\right)\right)=\coprod_{s \in S_{n} K} \mathbb{Z}\left(\operatorname{Mor}_{\text {Sub-G}}\left(-, G_{s}\right)\right)
$$

and that, for any generic coefficient system $M$, there is a $\mathbb{Z}(G)$-module cochain complex isomorphism

$$
C^{*}(K, M)=\operatorname{Hom}_{O_{G,(e)}}\left(c_{*}(K), M\right) .
$$

It follows from 2.3 that, for any subgroup $H$ of $G$,

$$
C^{*}(K, M)^{H}=\operatorname{Hom}_{O_{G, H}}\left(c_{*}(K), M\right) .
$$

We shall denote by $\bar{c}_{*}(K)$ the chain complex of functors from $\mathcal{K}_{G}$ to Ab such that, for any finite subcomplex $K^{\prime}$ of $K, \bar{c}_{*}(K)\left(K^{\prime}\right)$ is the ordinary cellular chain complex $C_{*}\left(K^{\prime}, \mathbb{Z}\right)$. If $f$ is a morphism of $\mathcal{K}_{G}$ then $\bar{c}_{*}(K)(f)=$ $C_{*}(f, \mathbb{Z})$. The restriction of $\bar{c}_{*}(K)$ to the category $\mathcal{K}(G)$ will be denoted by the same symbol. If $s$ is a cell of $K$ then $\bar{c}_{*}(K)(s)=C_{*}(K(s), \mathbb{Z})$. Let $\lambda: \mathcal{K}(e) \rightarrow \mathcal{K}(G)$ denote the natural inclusion. It is easy to see that

$$
\bar{c}_{n}(K) \lambda=\coprod_{s \in S_{n} K} \mathbb{Z}\left(\operatorname{Mor}_{\mathcal{K}(e)}(s,-)\right)
$$


and that, for any local coefficient system $L$, there is an isomorphism of $\mathbb{Z}(G)$-module cochain complexes

$$
C^{*}(K, L)=\operatorname{Hom}_{\mathcal{K}(e)}\left(\bar{c}_{*}(K), L\right) .
$$

Thus, for any subgroup $H$ of $G$ and any local coefficient system $L: \mathcal{K}(G) \rightarrow$ $\mathrm{Ab}$, there is an isomorphism of cochain complexes

$$
C^{*}(K, L)^{H}=\operatorname{Hom}_{\mathcal{K}(H)}\left(\bar{c}_{*}(K), L\right) .
$$

Proof of Proposition 1.6. This is an immediate consequence of 2.6 in the case where $W_{d}[G]^{\mathrm{op}}=\mathcal{K}(G), M=L$ and $C_{*}=\bar{c}_{*}(K)$.

As an immediate consequence of 2.5 , we obtain the following result.

2.7. Corollary. Assume that $H$ is a subgroup of $G$.

(i) Let $M$ be a G-generic coefficient system. Then there is an isomorphism $M[G / H] \rightarrow \operatorname{Hom}_{O_{G, H}}\left(O_{G}, M\right)$.

(ii) Let $K$ be a $G$-CW-complex and let $L$ be a local coefficient system on $K$. Then there is an isomorphism $L[G / H] \rightarrow \operatorname{Hom}_{\mathcal{K}(H)}(\mathcal{K}(G), L)$.

Corollary 1.13 yields the next result.

2.8. Corollary. Let $K$ be a $G$-CW-complex and let $H$ be a subgroup of $G$. Then there exists a natural equivalence of chain complexes of $G$ coefficient systems

$$
v_{*}(G / H): O_{G} \otimes_{O_{G, H}} c_{*}(K) \rightarrow c_{*}(K[G / H]) .
$$

Proof. This follows from the fact that, for any $G$-generic coefficient system $M$, there is an isomorphism

$$
\operatorname{Hom}_{O_{G}}\left(c_{*}(K[G / H]), M\right) \rightarrow \operatorname{Hom}_{O_{G, H}}\left(c_{*}(K), M\right) .
$$

Consider the $H$-CW-map $u=u(G / H, K): K \rightarrow K[G / H]$ described before Proposition 1.9. It follows from the proof of 1.9 that this map induces an isomorphism of cochain complexes

$$
w^{*}=w^{*}(G / H, M): C^{*}(K[G / H], M)^{G} \rightarrow C^{*}(K, M)^{H} .
$$

For every subgroup $J$ of $G$, the map $u$ induces a map $u^{J}: K^{J} \rightarrow K[G / H]^{J}$.

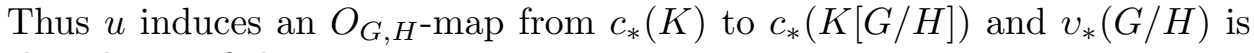
the adjoint of this map.

The following fact will be used in the proof of 1.16. We shall use the notation of 1.14 and 1.15. For any two subgroups $H, H^{\prime}$ of $G$, we shall denote by $p\left(H, H^{\prime}\right)$ the $G$-map $G /\left(H \cap H^{\prime}\right) \rightarrow G / H^{\prime}$ induced by the inclusion $H \cap H^{\prime} \subseteq H^{\prime}$.

2.9. Proposition. Let $K$ be a $G$-CW-complex and let $M$ be a generic coefficient system for $G$. Assume that $H$ is a subgroup of $G$ and that, for 
every $k$ in $K$, the map $M\left(p\left(H, G_{k}\right)\right): M\left(G / G_{k}\right) \rightarrow M\left(G /\left(H \cap G_{k}\right)\right)$ is an isomorphism. Then the map

$\phi^{*}(K, M)(G / H) z^{*}(K, M)(G / H): H_{G}^{*}(K[G / H], M) \rightarrow H_{G}^{*}(G / H \times K, M)$

is an isomorphism.

Proof. The $G$-set $S(G / H \times K)$ of all cells of $G / H \times K$ is equal to the $G$-set $G / H \times S(K)$. If $s$ is a cell of $K$ and $g$ is an element of $G$ then

$$
M \theta_{G}(K) p_{G / H}(g H, s)=M\left(G / G_{s}\right),
$$

$M \theta_{G}(G / H \times K)(g H, s)=M\left(G /\left(g H g^{-1} \cap G_{s}\right)\right)=M\left(G / g\left(H \cap G_{g^{-1} s}\right) g^{-1}\right)$, and $\varphi\left(M, p_{G / H}\right)(g H, s)$ is the composition of three isomorphisms:

$M\left(G / G_{s}\right) \rightarrow M\left(G / g^{-1} G_{s} g\right) \rightarrow M\left(G /\left(H \cap g^{-1} G_{s} g\right)\right) \rightarrow M\left(G / g H g^{-1} \cap G_{s}\right)$.

Now, the result follows from 1.15(iii).

Proof of Corollary 1.16. This result follows from 2.9 and from the facts that the functor $M$ is isomorphic to $H_{G}^{0}(-, M)=\mathcal{H}_{G}^{0}(G / G, M)$ and that the functor $\mathcal{I}_{G}^{0}(G / G, M)$ is constant because $(G / G)[G / H]=G / G$ whenever $H$ is a subgroup of $G$.

We shall now compare our constructions with the Illman transfer, which was defined in [7]. We shall denote by $O_{G, H}(K)$ the full subcategory of $O_{G, H}$ whose objects are of the form $G / G_{k}$, where $k \in K$. Let $\lambda_{H}: O_{G, H}(K) \rightarrow O_{G}$ be the natural inclusion and let $\omega_{H}: O_{G, H}(K) \rightarrow O_{H}$ be the functor such that $\omega_{H}\left(G / H^{\prime}\right)=H / H \cap H^{\prime}$. Assume that $M$ is a generic $G$-coefficient system, $N$ is a generic $H$-coefficient system and that there exists a natural transformation of functors $\Omega: N \omega_{H} \rightarrow M \lambda_{H}$. Then there exists the Illman transfer

$$
\tau^{*}(K, \Omega): H_{H}^{*}(K, N) \rightarrow H_{G}^{*}(K, M) .
$$

Immediately from the definition and from 1.6, it follows that $\tau^{*}(K, \Omega)$ is the composition of the map determined by $\Omega^{*}: C^{*}(K, N)^{H} \rightarrow C^{*}(K, M)^{H}$, which is induced by $\Omega$, and $i(H, G): \mathcal{I}_{G}^{*}(K, M)(G / H) \rightarrow H_{G}^{*}(K, M)$, which was described in 1.20 .

2.10. Example. Let $M$ be a generic $G$-coefficient system and let $H$ be a subgroup of $G$. Assume that $M$ satisfies the assumptions of 2.9. Let $I_{H}: O_{H} \rightarrow O_{G}$ be the natural functor such that, for every subgroup $H^{\prime}$ of $H, I_{H}\left(H / H^{\prime}\right)=G / H^{\prime}$. Consider the map $\Omega: M I_{H} \omega_{H} \rightarrow M \lambda_{H}$ such that, for every $k$ in $K, \Omega\left(G / G_{k}\right)=M\left(p\left(H, G_{k}\right)\right)^{-1}$. Then there exists the Illman transfer

$$
\tau^{*}(K, \Omega): H_{H}^{*}\left(K, M I_{H}\right)=H_{G}^{*}(G / H \times K, M) \rightarrow H_{G}^{*}(K, M)
$$

and the composition

$$
\tau^{*} \phi^{*}(K, M)(G / H): \mathcal{I}_{G}^{*}(K, M)(G / H) \rightarrow \mathcal{I}_{G}^{*}(K, M)(G / G)
$$


is the map $i(H, G)$ from 1.20 .

A $G$-generic coefficient system $M$ is naturally equivalent to the restriction of the functor $H_{G}^{0}(-, M)$ to the category $O_{G}$. Hence Corollary 1.15 implies that the functor $\gamma(i$,id $)$, where $\gamma$ is the functor described in 1.2, is naturally equivalent to a functor $\gamma_{0}: O_{G}^{\text {op }} \times\left(O_{G}^{\text {op }}, \mathrm{Ab}\right) \rightarrow\left(O_{G}^{\text {op }}, \mathrm{Ab}\right)$, defined in such a way that

$$
\gamma_{0}(G / H, M)\left(G / H_{1}\right)=\operatorname{Hom}_{O_{G}}\left(\mathbb{Z}\left(\operatorname{Map}_{G}\left(-, G / H_{1}[G / H]\right)\right), M\right)
$$

whenever $H, H_{1}$ are subgroups of $G$ and $M$ is a $G$-generic coefficient system. Recall that

$$
G / H_{1}[G / H]=\left(\left(G / H \times G / H_{1}\right) / G\right) \times G / H_{1} .
$$

The "classical" uniqueness theorem ([2], IV. 4,5) implies that there exists an isomorphism of cohomology theories

$$
r^{*}(-, M)(G / H): H_{G}^{*}\left(-, \gamma_{0}(G / H, M)\right) \rightarrow H_{G}^{*}(-[G / H], M)
$$

which is natural with respect to $M$ in $\left(O_{G}^{\mathrm{op}}, \mathrm{Ab}\right)$ and $G / H$ in $O_{G}$.

Let $\Gamma_{0}: O_{G}^{\mathrm{op}} \times\left(O_{G}^{\mathrm{op}}, \mathrm{Ab}\right) \rightarrow\left(O_{G}^{\mathrm{op}}, \mathrm{Ab}\right)$ be the functor such that, for any subgroup $H$ of $G$ and for any $G$-generic coefficient system $M, \Gamma_{0}(G / H, M)$ is the restriction of the functor $H^{0}(G / H \times-, M)$ to $O_{G}$. Then

$$
\Gamma_{0}(G / H, M)\left(G / H_{1}\right)=\operatorname{Hom}_{O_{G}}\left(\mathbb{Z}\left(\operatorname{Map}_{G}\left(-, G / H \times G / H_{1}\right)\right), M\right)
$$

and there is an isomorphism of cohomology theories

$$
\mathrm{r}_{0}^{*}(-, M)(G / H): H_{G}^{*}\left(-, \Gamma_{0}(G / H, M)\right) \rightarrow \mathcal{H}_{G}^{*}(-, M)(G / H)
$$

which is natural with respect to $M$ in $\left(O_{G}^{\mathrm{op}}, \mathrm{Ab}\right)$ and $G / H$ in $O_{G}$.

The $G$-map $\phi\left(G / H, G / H_{1}\right)$ determines a natural transformation of functors $\phi_{0}: \gamma_{0} \rightarrow \Gamma_{0}$. As a consequence of 1.14 and 1.15 we obtain the following result.

2.11. Corollary. Let $\phi_{0}^{*}: H_{G}^{*}\left(-, \gamma_{0}(-,-)\right) \rightarrow H_{G}^{*}\left(-, \Gamma_{0}(-,-)\right)$ be the natural transformation which is induced by $\phi_{0}$. Then $\phi^{*}\left(z^{*}\right)^{-1} \mathrm{r}^{*}=\mathrm{r}_{0}^{*} \phi_{0}^{*}$ where $\phi^{*}, z^{*}$ are the natural transformations described in 1.15.

Proof. This follows from the fact that, by [2], Ch. IV.4, 5,

$$
\mathrm{r}_{0}^{*}(K, M)(G / H) \phi_{0}^{*}(K, M)(G / H)=H^{*}(\phi(G / H, K), M) \mathrm{r}^{*}(K, M)(G / H)
$$

whenever $H$ is a subgroup of $G, K$ is a $G$-CW-complex and $M$ is a generic $G$-coefficient system.

We shall now prove Propositions 1.18 and 1.19 . Let $K$ be a $G-\mathrm{CW}$ complex. Then we have the Hecke functor chain complex $\widetilde{c}_{*}(K): \mathbb{H}_{G}^{\mathrm{op}} \rightarrow \mathrm{Ab}_{\mathrm{c}}$ such that, for any subgroup $H$ of $G$,

$$
\widetilde{c}_{*}(K)(G / H)=\operatorname{Hom}_{\mathbb{Z}(G)}\left(\mathbb{Z}(G / H), C_{*}(K, \mathbb{Z})\right)=C_{*}(K, \mathbb{Z})^{H} .
$$


From the fact that $c_{*}(K)$ is a direct sum of representable $O_{G}^{\text {op }}$ functors and $\widetilde{c}_{*}(K)$ is the direct sum of the analogous representable $\mathbb{H}_{G}^{\text {op }}$ functors it follows that $\widetilde{c}_{*}(K)$ is the left Kan extension of $c_{*}(K)$. Assume that $T$ is a Hecke functor. Then there is an isomorphism of cochain complexes

$$
\operatorname{Hom}_{O_{G}}\left(c_{*}(K), T i\right) \rightarrow \operatorname{Hom}_{\mathbb{H}_{G}}\left(\widetilde{c}_{*}(K), T\right),
$$

which is natural in $K$ and $T$. This isomorphism follows from the formal properties of left Kan extensions. If $X$ is a finite $G$-set, then

$$
H_{G}^{*}(X, T)=H_{G}^{0}(X, T) \cong \operatorname{Hom}_{\mathbb{H}_{G}}\left(\operatorname{Hom}_{\mathbb{Z}(G)}(\iota(-), \mathbb{Z}(X)), T\right) .
$$

Proof of Proposition 1.18. Assume that $T$ is a Hecke functor. Let $T^{\prime}$ be the contravariant functor from $\mathbb{Z}(G)$-Mod ${ }^{\text {op }}$ to Ab such that

$$
\begin{aligned}
T^{\prime}(A) & =\operatorname{Hom}_{\mathbb{H}_{G}}\left(\operatorname{Hom}_{\mathbb{Z}(G)}(\iota(-), A), T\right), \\
T^{\prime}(f) & =\operatorname{Hom}_{\mathbb{H}_{G}}\left(\operatorname{Hom}_{\mathbb{Z}(G)}(\iota(-), f), \operatorname{id}_{T}\right),
\end{aligned}
$$

whenever $A$ is a $\mathbb{Z}(G)$-module and $f$ is a $\mathbb{Z}(G)$-module homomorphism. This means that $T^{\prime}$ is a right Kan extension of $T$ along the functor $\iota$. It is obvious that $T^{\prime} \iota=T$. We define $\Gamma$ by

$$
\begin{aligned}
\Gamma(G / H, T)\left(G / H_{1}\right) & =T^{\prime}\left(\mathbb{Z}(G / H) \otimes \mathbb{Z}\left(G / H_{1}\right)\right), \\
\Gamma(G / H, T)(f) & =T^{\prime}(\operatorname{id} \otimes \mathbb{Z}(f)),
\end{aligned}
$$

whenever $H, H_{1}$ are subgroups of $G$ and $T$ is a Hecke functor. If $f^{\prime \prime}$ : $\mathbb{Z}(G / H) \rightarrow \mathbb{Z}\left(G / H^{\prime \prime}\right)$ is a $\mathbb{Z}(G)$-module homomorphism and if $\varrho: T \rightarrow T_{0}$ is a natural transformation of Hecke functors, then

$$
\Gamma\left(f^{\prime \prime}, \varrho\right)\left(G / H_{1}\right)=\varrho^{\prime}\left(f^{\prime \prime} \otimes \mathrm{id}\right) .
$$

(One can simply observe that the category $\mathbb{H}_{G}$ can be defined either in terms of just the orbits $G / H$ or in terms of finite $G$-sets. The two definitions give the same category of additive functors into $\mathrm{Ab}$. With the definition in terms of finite $G$-sets, one can define $\Gamma(G / H, T)$ to be $T(G / H \times(-))$.)

It follows from the definition that there is an isomorphism

$$
H_{G}^{*}\left(G / H \times G / H_{1}, T\right)=H_{G}^{0}\left(G / H \times G / H_{1}, T\right) \cong H_{G}^{*}\left(G / H_{1}, \Gamma(G / H, T)\right),
$$

which is natural with respect to $G / H$ and $G / H_{1}$ in $O_{G}$ and $T$ in $\left(\mathbb{H}_{G}^{\mathrm{op}}, \mathrm{Ab}\right)$. Thus there exists an isomorphism of cohomology theories

$$
\psi^{*}(G / H, T): H_{G}^{*}(-, \Gamma(G / H, T)) \rightarrow H_{G}^{*}(G / H \times(-), T),
$$

which is natural with respect to $G / H$ in $O_{G}$ and $T$ in $\left(\mathbb{H}_{G}^{\mathrm{op}}, \mathrm{Ab}\right)$.

Proof of Proposition 1.19. Consider the functors $D_{1}, D_{2}: \mathbb{H}_{G} \times$ $O_{G} \rightarrow \mathbb{Z}(G)$-Mod defined by

$$
\begin{aligned}
& D_{1}\left(G / H, G / H_{1}\right)=\mathbb{Z}(G / H) \otimes \mathbb{Z}\left(G / H_{1}\right) \cong \mathbb{Z}\left(G / H \times G / H_{1}\right), \\
& D_{2}\left(G / H, G / H_{1}\right)=\left(\left(\mathbb{Z}(G / H) \otimes \mathbb{Z}\left(G / H_{1}\right)\right) \otimes_{\mathbb{Z}(G)} \mathbb{Z}\right) \otimes \mathbb{Z}\left(G / H_{1}\right)
\end{aligned}
$$




$$
\cong \mathbb{Z}\left(\left(G / H \times G / H_{1}\right) / G\right) \otimes \mathbb{Z}\left(G / H_{1}\right) \cong \mathbb{Z}\left(G / H_{1}[G / H]\right) .
$$

If $f^{\prime \prime}: \mathbb{Z}(G / H) \rightarrow \mathbb{Z}\left(G / H^{\prime \prime}\right)$ is a $\mathbb{Z}(G)$-module homomorphism and $f_{0}$ : $G / H_{1} \rightarrow G / H_{2}$ is a $G$-map, then $D_{1}\left(f^{\prime \prime}, f_{0}\right)=f^{\prime \prime} \otimes \mathbb{Z}\left(f_{0}\right)$ and $D_{2}\left(f^{\prime \prime}, f_{0}\right)=$ $\left(\left(f^{\prime \prime} \otimes \mathbb{Z}\left(f_{0}\right)\right) \otimes \mathrm{id}\right) \otimes \mathbb{Z}\left(f_{0}\right)$. The $\mathbb{Z}(G)$-module homomorphisms

$$
\mathbb{Z}\left(\phi\left(G / H, G / H_{1}\right)\right): \mathbb{Z}\left(G / H \times G / H_{1}\right) \rightarrow \mathbb{Z}\left(G / H_{1}[G / H]\right)
$$

induce a natural transformation $\zeta_{0}: D_{1} \rightarrow D_{2}$ of functors from $\mathbb{H}_{G} \times O_{G}$ to $\mathbb{Z}(G)$-Mod. It follows from the definitions that there are isomorphisms

$$
\begin{aligned}
\gamma^{\prime}(G / H, T)\left(G / H_{1}\right) & \cong \operatorname{Hom}_{\mathbb{Z}(G)}\left(\mathbb{Z}\left(G / H \times G / H_{1}\right), \mathbb{Z}\right) \otimes T\left(G / H_{1}\right) \\
& \cong \operatorname{Hom}_{\mathbb{H}_{G}}\left(\operatorname{Hom}_{\mathbb{Z}(G)}\left(\iota(-), D_{2}\left(G / H, G / H_{1}\right)\right), T\right)
\end{aligned}
$$

which are natural with respect to $G / H$ in $\mathbb{H}_{G}, G / H_{1}$ in $O_{G}$ and $T$ in $\left(\mathbb{H}_{G}^{\mathrm{op}}, \mathrm{Ab}\right)$. Let $\zeta$ be the natural transformation from $\gamma^{\prime}$ to $\Gamma$ induced by $\zeta_{0}$. Then $\zeta$ determines a natural transformation $\zeta^{*}: \mathcal{I}_{G}^{*} \rightarrow \Gamma^{*}$ such that $\psi^{*} \zeta^{*}=\phi^{*}$.

Now, we shall prove Lemma 1.24. Let $K$ be a $G$-CW-complex. Assume that $F$ is a $G$-set of subgroups of $G$ which contains the group $G$ and all subgroups of the form $G_{k}$ where $k \in K$. It is well known that, for any generic coefficient system $M$ and for any Hecke functor $T$,

$$
\begin{aligned}
\operatorname{Hom}_{O_{G}}\left(c_{*}(K), M\right) & =\operatorname{Hom}_{O(F)}\left(c_{*}(K), M\right), \\
\operatorname{Hom}_{\mathbb{H}_{G}}\left(\widetilde{c}_{*}(K), T\right) & =\operatorname{Hom}_{\mathbb{H}(F)}\left(\widetilde{c}_{*}(K), T\right),
\end{aligned}
$$

where $\mathbb{H}(F)$ is the full subcategory of $\mathbb{H}_{G}$ whose objects are all orbits $G / H$ such that $H \in F$. The statement (i) of 1.24 is a well known consequence of the existence of the spectral sequence which is described in [2], Ch. I.10. The statement (ii) can be proved in a similar way.

Proof of Lemma 1.24(ii). For any natural number $n$, the functor $\widetilde{c}_{n}(K, R)=\widetilde{c}_{n}(K) \otimes R$ is a projective object in the category $(\mathbb{H}(F), R$-Mod), because it is a sum of representable functors tensored with $R$. Thus there exists a spectral sequence

$$
\operatorname{Ext}_{\mathbb{H}(F)}^{p}\left(\widetilde{h}_{q}(K, R), T\right) \Rightarrow H_{G}^{p+q}(K, T),
$$

where $\operatorname{Ext}_{\mathbb{H}(F)}^{p}$ is the $n$th derived functor of the functor $\operatorname{Hom}_{\mathbb{H}(F)}$ of natural transformations of functors from $\mathbb{H}(F)^{\text {op }}$ to $R$-Mod and $\widetilde{h}_{q}(K, R)$ is the $q$ th homology functor of the chain complex $\widetilde{c}_{*}(K, R)$. For any subgroup $H$ of $G$

$$
\widetilde{h}_{q}(K, R)(G / H)=H_{q}\left(\operatorname{Hom}_{\mathbb{Z}(G)}\left(\mathbb{Z}(G / H), C_{*}(K, R)\right)\right)=H_{q}\left(C_{*}(K, R)^{H}\right) .
$$

It follows from the assumptions that $\widetilde{h}_{q}(K, R)=(0)$ for $q>0$ and

$$
\widetilde{h}_{0}(K, R)=\widetilde{h}_{0}(G / G, R)=\operatorname{Mor}_{\mathbb{H}(F)}(-, G / G) \otimes R
$$


is a projective object in the category of additive functors $\left(\mathbb{H}(F)^{\mathrm{op}}, R\right.$-Mod). Hence $H_{G}^{*}(K, T)=\operatorname{Hom}_{\mathbb{H}(F)}\left(\operatorname{Mor}_{\mathbb{H}(F)}(-, G / G) \otimes R, T\right)=T(G / G)$.

Other applications of the Hecke structure on the Bredon cohomology theory will be presented in [11] and [12].

\section{References}

[1] M. Auslander, Representation theory of artin algebras I, Comm. Algebra 1 (3) (1974), 177-268.

[2] G. E. Bredon, Equivariant Cohomology Theory, Lecture Notes in Math. 34, Springer, 1967.

[3] —, Introduction to Compact Transformation Groups, Academic Press, 1972.

[4] K. S. Brown, Cohomology of Groups, Springer, 1982.

[5] T. tom Dieck, Equivariant homology and Mackey functors, Math. Ann. 206 (1973), $67-78$.

[6] J. A. Green, Axiomatic representation theory for finite groups, J. Pure Appl. Algebra 1 (1971), 41-77.

[7] S. Illman, Equivariant singular homology and cohomology I, Mem. Amer. Math. Soc. 156 (1975).

[8] S. Jackowski and J. E. McClure, Homotopy decomposition of classifying spaces via elementary abelian subgroups, Topology, to appear.

[9] B. Mitchell, Rings with several objects, Adv. in Math. 8 (1972), 1-161.

[10] J. Słomińska, Equivariant Bredon cohomology of classifying spaces of families of subgroups, Bull. Acad. Polon. Sci. Sér. Sci. Math. 28 (1980), 503-508.

[11] - Finiteness conditions in Bredon cohomology, J. Pure Appl. Algebra, to appear.

[12] -, Smith theory and quasi-periodicity in Bredon cohomology, in preparation.

[13] S. Waner, A generalization of cohomology of groups, Proc. Amer. Math. Soc. 85 (1982), 469-474.

[14] —, Mackey functors and G-cohomology, ibid. 90 (1984), 641-648.

[15] T. Yoshida, On G-functors. I, Hokkaido Math. J. 9 (1980), 222-257.

[16] -, On G-functors. II, J. Math. Soc. Japan 35 (1983), 179-190.

INSTITUTE OF MATHEMATICS

NICHOLAS COPERNICUS UNIVERSITY

CHOPINA $12 / 18$

87-100 TORUŃ, POLAND

Received 6 October 1988;

in revised form 7 February 1990, 15 October 1990,

and 11 April 1991 